\title{
Experimental and Numerical Study of Laminar and Turbulent Base Flow on a Spherical Capsule
}

\author{
Matthew MacLean* \\ Erik Mundy* \\ Timothy Wadhams \\ Michael Holden ${ }^{\dagger}$ \\ CUBRC, Aerothermal/Aero-optics Evaluation Center, Buffalo, NY, 14225 \\ Michael Barnhardt ${ }^{\ddagger}$ \\ Graham Candler ${ }^{\S}$ \\ Department of Aerospace Engineering and Mechanics \\ University of Minnesota, Minneapolis, MN 55455
}

\begin{abstract}
A review is presented of ground test measurements on a spherical capsule model with aftbody measurements of heating and pressure for a range of enthalpies and Reynolds numbers to obtain datasets of fundamental validation data for CFD codes and to develop a database for design-of-experiment of future studies. These measurements have been made using several sting mounting arrangements and significant differences in heating of up to $100 \%$ have been observed. Increasing relative heat transfer with increasing Reynolds numbers has been observed which may be indicative of transitional behavior on the aftbody. The relative heating on the aftbody has also been found to be exceptionally sensitive to the flow total enthalpy. Measurements of jet and cavity interaction on the otherwise smooth aftbody have also been explored and a non-dimensional correlation developed for design of future test articles. Finally, comparisons with the US3D code using a DES methodology have been made with the data and generally good agreement to within one standard deviation has been found. The most significant limitations have been found in modeling transition and in matching heating and pressure locally near separation points.
\end{abstract}

\section{Introduction}

$\mathrm{T}$ he recent resurgence of interest in spherical capsule geometries for reentry has highlighted the need for additional experimental datasets and code validation on this type of geometry. The Orion crew vehicle selected for use as the reentry configuration in NASA's current Constellation program has generated additional concerns in specifying the aerothermal heat load to a spherical capsule vehicle since it is significantly larger than any previous vehicle of this shape. This increased size automatically implies higher Reynolds number scales and an increased role of transition and turbulence in the vehicle characterization. One particular area of concern is the limited information available on the aftbody of the vehicle in terms of base heating and wake region structure. While the base region of the vehicle experiences significantly lower heating that the forebody, it is where most of the sensitive areas of the OML are located, such as egress hatches, communications antennae, and reaction control system (RCS) thrusters to control the vehicle's attitude during reentry. This base region of the vehicle is difficult to interrogate experimentally because of interference from the sting. The experimental datasets presented here provide validation data for aftbody heating and pressure distributions in the regime of transitional and turbulent flows with several candidate sting arrangements to assess the influence of each geometry on the aftbody flowfield. Additionally, the effects of jet interaction have been explored by making measurements on a representative arrangement involving a single jet, a single cavity, and the surrounding aftbody surface.

\footnotetext{
* Senior Research Scientist, AIAA Member.

†AAEC Program Manager, AIAA Fellow.

$\ddagger$ Graduate Research Assistant, now Research Scientist at ELORET Corp, AIAA Member.

§Professor, AIAA Fellow.
} 
Aftbody flowfield predictions in massively separated regions of hypersonic flow using conventional Reynolds Averaged Navier-Stokes (RANS) models have not been sufficiently validated, although the use of RANS in these regions is generally met with skepticism by the CFD community. Using the experimental database made available during this program, preliminary validation with a detached-eddy simulation (DES) technique using the US3D code developed by the University of Minnesota has been performed under separate funding and some results will be shown that indicate promise for such a technique to be employed in next-generation vehicle design.

\section{Experimental Facility Background}

Currently, CUBRC operates the 48” reflected shock tunnel, the LENS-I and LENS-II reflected shock tunnels, and the LENS-XX expansion tunnel ${ }^{2}$. The reflected shock tunnel uses shocks to heat and pressurize a stagnant test gas to high enthalpy levels. This test gas may then be expanded through a converging-diverging nozzle in a manner similar to a blowdown facility to produce a hypervelocity test flow. Expansion tunnels like LENS-X also operate as short duration facilities, but an expansion tunnel produces a high enthalpy flow without the need to first stagnate the test gas. In an expansion tunnel, the high enthalpy gas is generated in two stages. In the first stage, the test gas in the driven tube is compressed and heated by a shock, but the moving flow behind the incident shock is not immediately brought to a halt by a reflected shock as in a reflected shock tunnel. Secondly, additional energy is added to this moving gas by an unsteady expansion in the acceleration segment of the tunnel to produce a low static temperature, high velocity test gas which is then isentropically expanded in a diverging nozzle to achieve a high enthalpy test flow at a thermodynamic state like that of a flight condition. The result is a freestream flow that is free of frozen, dissociated chemical contamination that can plague reflected shock facilities at enthalpies exceeding $10 \mathrm{MJ} / \mathrm{kg}$. While the useable test times from an expansion tunnel are generally shorter than those from a reflected shock tunnel, the clean, very high enthalpy flows that an expansion tunnel can generate provides a unique testing capability. A notional range of velocity and altitude duplication capabilities for all the LENS facilities is given using air test gas in Fig. 1.

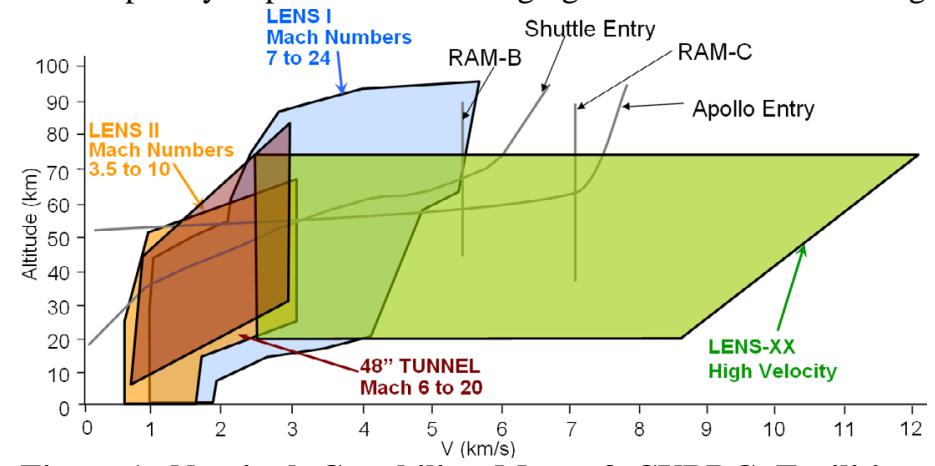

Figure 1. Nominal Capability Map of CUBRC Facilities to Duplicate Freestream Conditions in Air

The LENS reflected shock-tunnel facilities were developed primarily to study the full-scale, hypervelocity flow physics of interceptors and air-breathing engine configurations. The scale and flow duplication capabilities of LENS are such that these vehicles can be studied at their full scale, inclusive of effects such as transition to turbulence, turbulent mixing from cross-flow jets and thrusters, duplicated flow chemistry and other effects that are difficult or impossible to simulate at cold-flow or sub-scale conditions. Besides aerothermal measurements, extensive studies in this facility have been made using non-intrusive diagnostics such as aero-optic and aero-acoustic measurements, including recent work with tunable laser-diode diagnostics ${ }^{3}$. The capabilities of LENS-I duplicate the flight conditions of interceptors and scramjet engines from Mach 7 to 15 (with Reynolds number matching to Mach 22), while the LENS-II facility complements it in such a way that this capability is seamlessly extended down to Mach 3.5 at sea level density.

\section{Numerical and Physical Modeling}

All ground test studies in the LENS facilities are extensively calibrated and validated with numerical tools. For most test programs at CUBRC, the primary CFD tool used in the validation is the DPLR code provided by NASA Ames Research Center. DPLR is a multi-block, structured, finite-volume code that solves the reacting NavierStokes equations including finite rate chemistry and finite rate vibrational non-equilibrium effects. This code is based on the data-parallel line relaxation method ${ }^{6}$ and implements a modified (low dissipation) Steger-Warming flux splitting approach $^{7,8}$. It employs several common RANS turbulence models that are used widely in the community.

In the study of aftbody flows which are dependent on large-scale separation, however, the validity of the RANS models are in doubt and the complex gridding requirements makes these problems difficult or impossible to adequately resolve using a fully-matched, structured block topology. Therefore, the US3D ${ }^{9,10}$ code was employed with a Detached-Eddy Simulation (DES) capability to perform the numerical comparisons with the data since this code is a better choice for this type of flow. The DES modification provides an enhancement to the turbulence 
modeling that creates a large-eddy simulation like behavior for large-scale turbulence and reduces to RANS behavior only in regions of small-scale turbulence such as immediately adjacent to solid walls. The DES model is inherently three-dimensional and unsteady, so comparisons to the data can be made either in instantaneous or timeaveraged senses using this physically "better" model of turbulence in the separated region.

US3D is a parallel, finite-volume, unstructured code developed at the University of Minnesota and much of its technical heritage is shared with NASA's structured DPLR code. In particular, the code employs an efficiently parallelized form of implicit line relaxation inside boundary layers with a point implicit method used elsewhere ${ }^{10}$. US3D uses numerous physical models and numerical options developed primarily for the solution of hypervelocity, non-equilibrium flows. In this work, the convective fluxes are treated with the Modified Steger-Warming scheme ${ }^{7}$ and computed via a 2nd order accurate TVD approach. Viscous fluxes are also $2^{\text {nd }}$ order and use a weighted leastsquares approach to reconstruct gradients. Contrary to what is typically found in literature regarding the computation of unsteady, time-accurate flows, US3D's temporal discretization is only $1^{\text {st }}$ order accurate. The time step is therefore limited to ensure that it does not exceed the smallest convective time scale present in the unsteady flow regions. The temperatures experienced during the tests considered here are insufficient to dissociate the gas and a perfect gas model for air is therefore used in all cases. Laminar diffusion coefficients are computed using a combination of Blottner curve fits for viscosity, an Eucken relation for thermal conductivity, and, for mass diffusion, a constant Lewis number of 1.4.

For cases in which the flow can reasonably be deemed transitional or turbulent, we use the one-equation Spalart-Allmaras (S-A) turbulence mode ${ }^{11}$ with compressibility corrections due to Catris and Aupoix ${ }^{12}$. While data from some of the experimental data clearly show transitional behavior in the windside boundary layers, no effort is made here to model transitional effects. Rather, our focus is on the massively separated flow of the aftbody. The deficiencies of this approach are largely localized to the transitional regions themselves, a fact which will be evident during the presentation of results. The turbulent, separated regions of the flow are modeled using the DES modification to the S-A model proposed by Spalart ${ }^{13}$. The methods used herein have been presented and validated in several other studies ${ }^{14}$.

\section{Capsule Design and Model Parameters}

The geometry selected for the study is a scaled Orion/Apollo OML shape based on a 10-inch primary diameter. The dimensions of the flowlines of the spherical capsule model are given in Fig 2. The origin of the coordinate system occurs at the imaginary point where the angled aftbody would meet if it was sharp. Axial positions on the capsule are measured forward from this point, with larger values occurring more forward on the capsule. The solid body CAD model geometry is shown in Fig 3, which highlights the main features and components of the design. The main components are the forebody and aftbody, broken near the peak of the shoulder to allow access to the inside for installation of instrumentation and routing of wiring. In addition, several inserts were machined into the aftbody to allow various attachment mechanisms. These are the traditional center-mount, split (forked) mount to allow flow on the symmetry plane to pass by the sting unimpeded, and blade and jet mount on the windward plane. Also, inserts for a single pitch jet and a single roll jet were machined in the aftbody. These inserts allowed for maximum flexibility in designing the experiments with several mounting arrangements, which were conducted in several phases.

For the smooth body test phases with different mounting schemes, three choices have been evaluated and compared using the surface pressure and heat transfer instrumentation. The basic dimensions of each sting are given in Fig 4. Note that the round and diamond stings are both mounted at the center of the capsule aftbody using the same mount insert location, but are different in shape (and potentially influence) on the aftbody flowfield dynamics. Pictures of the instrumented model are shown in Fig 5 installed in the facility with each sting. The model was located at nominally the same position in the test core of the facility with each configuration.

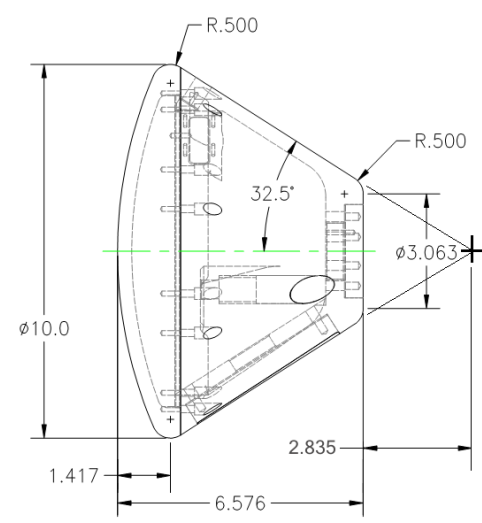

Figure 2. Major Dimensions of Spherical Capsule Model

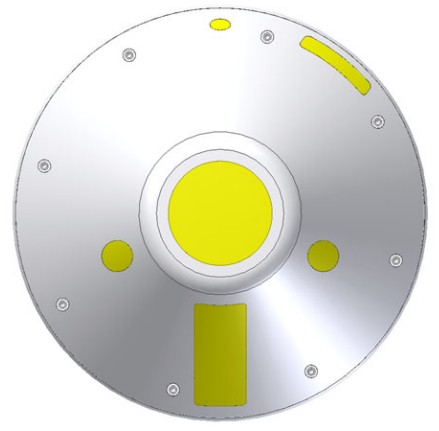

Figure 3. Solid Body CAD Model of Spherical Capsule Showing Inserts and Mounting Options 


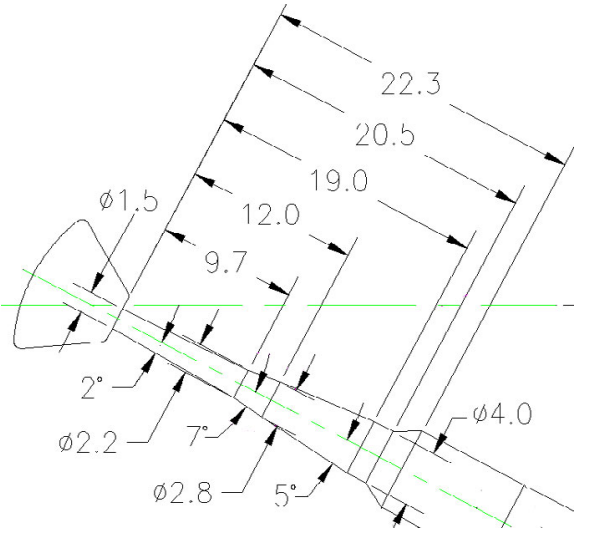

(a) Round Sting

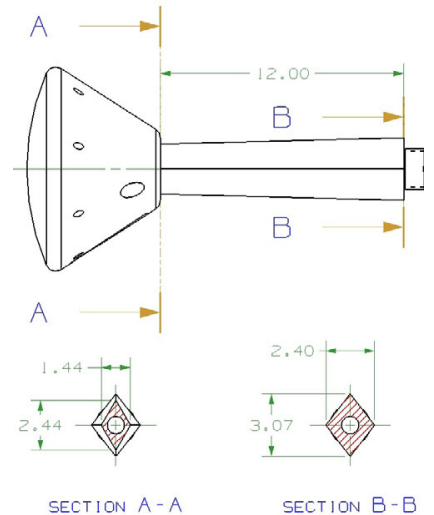

(b) Diamond Sting
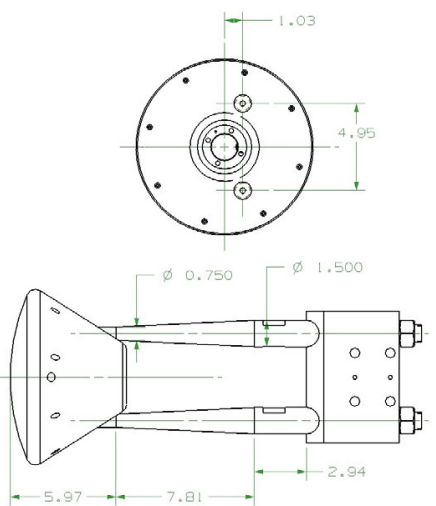

(c) Split Sting

Figure 4. Major Dimensions of Sting Arrangements (all dimensions in inches)

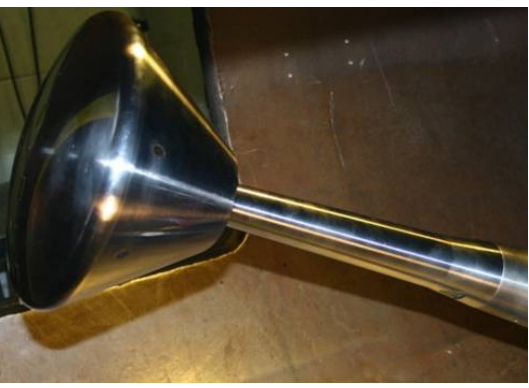

(a) Round Sting

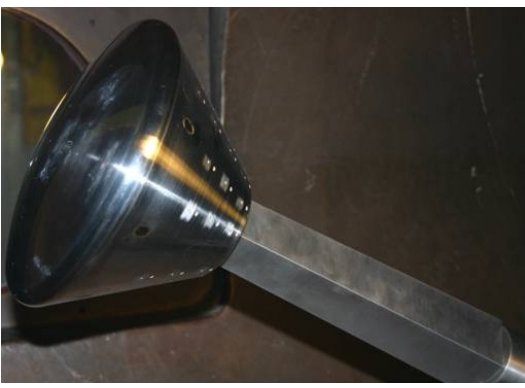

(b) Diamond Sting

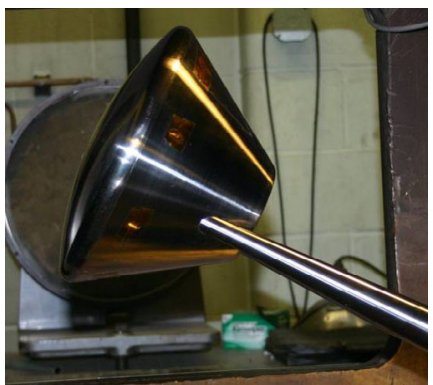

(c) Split Sting

Figure 5. Capsule Installed in Facility for Different Sting Mounts

Instrumentation is a central feature of any aerothermal model and this capsule model is instrumented with a total of 85 surface sensors. The model contains a total of 38 piezoelectric pressure gages and 47 heat transfer gages, inclusive of all inserts and optional measurements. The forebody instrumentation is limited to provide only basic confirmation of the test conditions. Four Medtherm coaxial thermocouple gages were placed on the centerline of the heat shield with one additional 0.040 " thin-film gage at each shoulder (six sensors total). The remaining sensors are 0.125" diameter thin-film and PCB piezoelectric pressure gages arrayed in rays along the aftbody. The instrumentation map is shown in Fig 6, with heat flux gages shown in red and pressure taps shown in blue. The

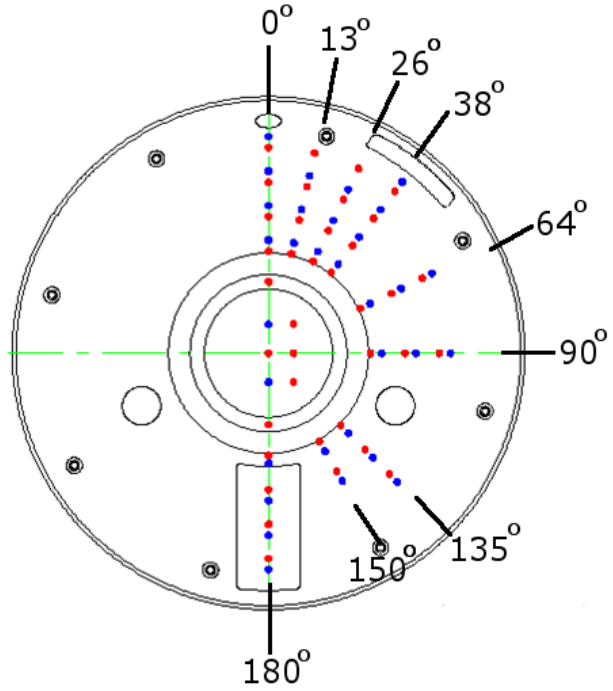

\section{$\bullet \triangle$ PRESSURE TAP \\ - HEAT TRANSFER GAGE}

Figure 6. Surface Heat Transfer and Pressure Instrumentation for Spherical Capsule Model 
instrumentation on the aftbody has been loosely arrayed in angular rays which are labeled in the figure to communicate the nomenclature employed to analyze the data in later sections. An understanding of this naming convention will be important in reading onward. All runs have been made at $28^{\mathrm{O}}$ angle of attack, near the peak $\mathrm{L} / \mathrm{D}$ for this shape. For sting mounting studies, the model was oriented such that the $180^{\circ}$ ray of instrumentation is most wind-ward. The flow in this region is attached since this ray is nearly parallel to the flow at this angle of attack. The most leeward ray is the $0^{\mathrm{O}}$ ray. Flow over these gages and rays near it are assumed to be separated for all conditions.

\section{Predicted and Measured Time Establishment of Wake Region}

Time establishment in the short duration facility is critical to the success of the experiment. While flow over the forebody establishes quite quickly, the wake takes much longer and can severely constrain short duration facilities if care is not taken. It is necessary to average the fullyestablished turbulent data over many flow lengths to obtain good quality results for code validation purposes. Based on results obtained from DES simulations of this type of problem, it was judged that 30-35 flow times (based on the model diameter) are sufficient. This means that it is necessary to get 30 flow times of data after the transient time-establishment process is completed.

A sample time history trace is considered in Fig 7 for a typical heat transfer gage on the leeward face of the aftbody near the center of the

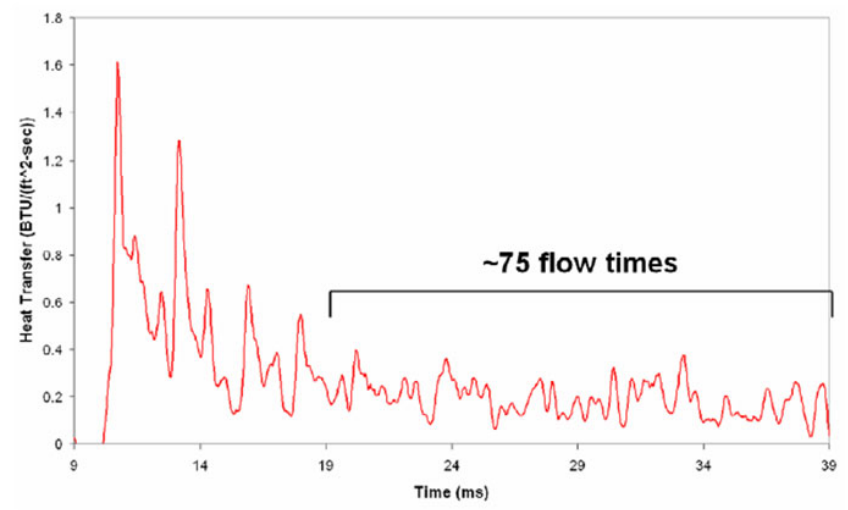

Figure 7. Typical Heat Transfer trace on Base Region of Capsule Showing Transient Start-up Process and Steadystate Averaging Time

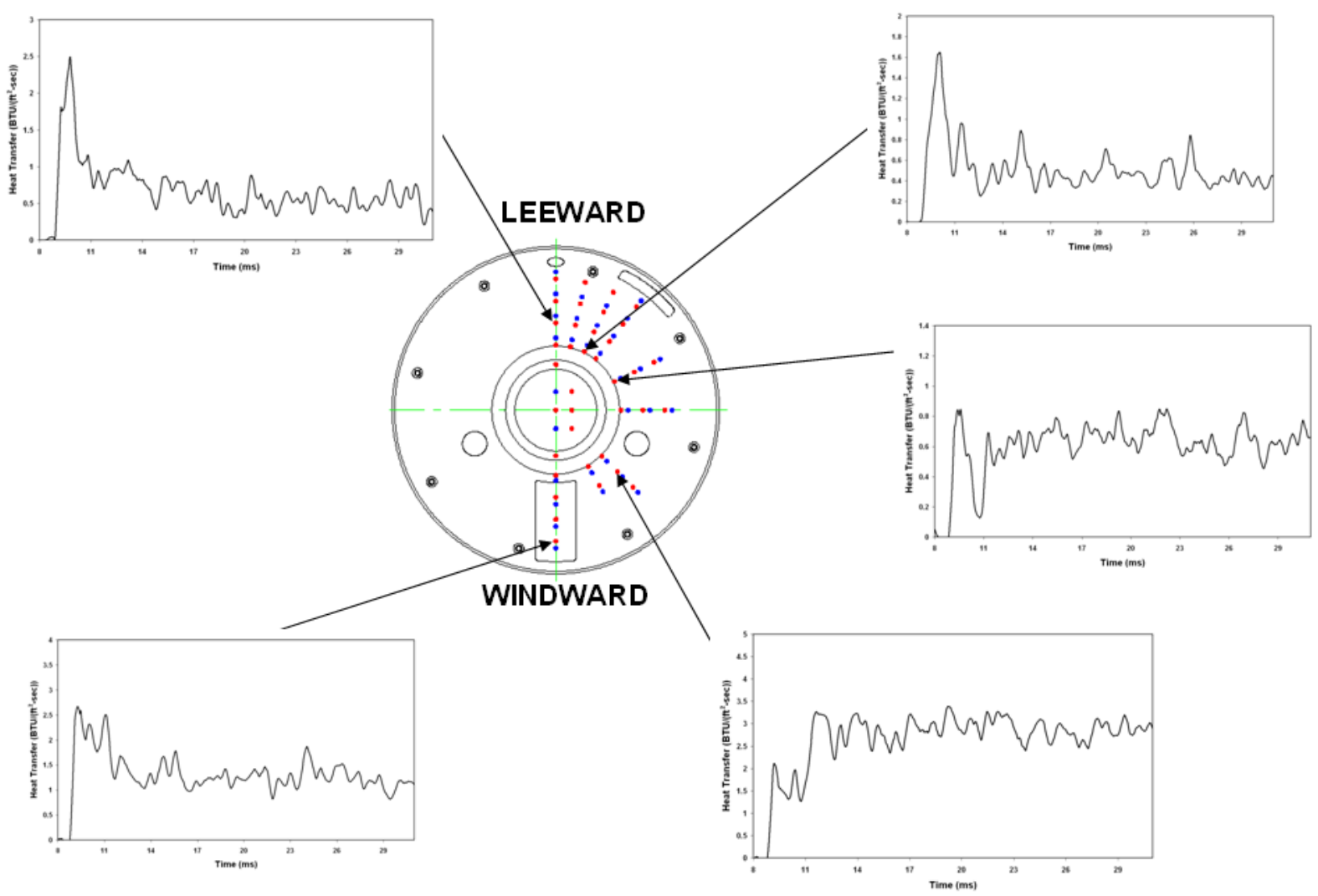

Figure 8. Typical Heat Transfer Traces at Different Points in the Base Region of the Capsule with Round Sting Showing Start-up Process and Steady-state Character 
separated region. It was found that the surface pressure establishes faster on the aftbody so the heat transfer measurement is the limiting constraint. We note that the character of the data is somewhat transitional for this case, so we do not expect the signals to be constant. They vary in time with statistical frequency based on the transitional instability and/or turbulent eddy fluctuations at that location but are considered fully-established in that, over a sufficiently long enough average, the time-averaged level is obtained. At this run condition, approximately 75 flow lengths of data are available. The total enthalpy of the flow was varied somewhat for different runs during this study, but the experiments always had 50 or more flow lengths with which to average over.

Figure 8 shows typical time history traces taken at random locations in the wake. The traces are generally similar to the typical traces shown in the previous figure. It is noted that, except for the most windward sensors, the time establishment seems to occur at about the same rate over the entire aftbody rather than propagating from one side to the other as one might initially expect. It also appears that the transient heating on the wake is rather benign, peaking for most gages at the start of the run and then smoothly decaying to the nominal steady-state signal level. The profile of the transient start-up process is somewhat position-dependent, but we did not observe any indications that the wake was experiencing any dramatic transients during the establishment period. It seems to be more a case of "filling-in."

\section{Study of Transition from Laminar to Turbulent Flow for Attached and Separated Aftbody Regions}

Before analyzing comparisons between the various sting mounting schemes, it is useful to analyze the character of the data for several Reynolds numbers at selected points on the model using the data from several runs. For attached flows, previous work has demonstrated that the thin-film gages can measure the transient spikes due to intermittency as the onset and turbulent break-down occurs ${ }^{16}$. In fact, our work has shown that the thin-films can often be used as an indicator of transition onset by computing the standard deviation of the measured signal. A sharp increase in the standard deviation value is typically observed sooner than the time-averaged heat transfer actually starts to noticeably rise ${ }^{17}$. Figure 9 shows that the transition process on the windward plane of the capsule looks very similar to what occurs on a cone. In this image, flow moves from right to left at the three Reynolds numbers shown. For the lower right-hand image, the flow is nominally laminar for each condition, although the highest Reynolds number case shows the first indications of the noisy character that precedes intermittency. In this study and in all others where we have made smooth body heating transfer measurements on a spherical capsule at angle of attack ${ }^{18}$, we have never seen a case where the smooth body heating remains turbulent in this region even if it was turbulent on the forebody shoulder ahead of the strong expansion. In the next trace (lower center), the highest Reynolds number shows a significantly intermittent behavior with the middle condition beginning to show the first

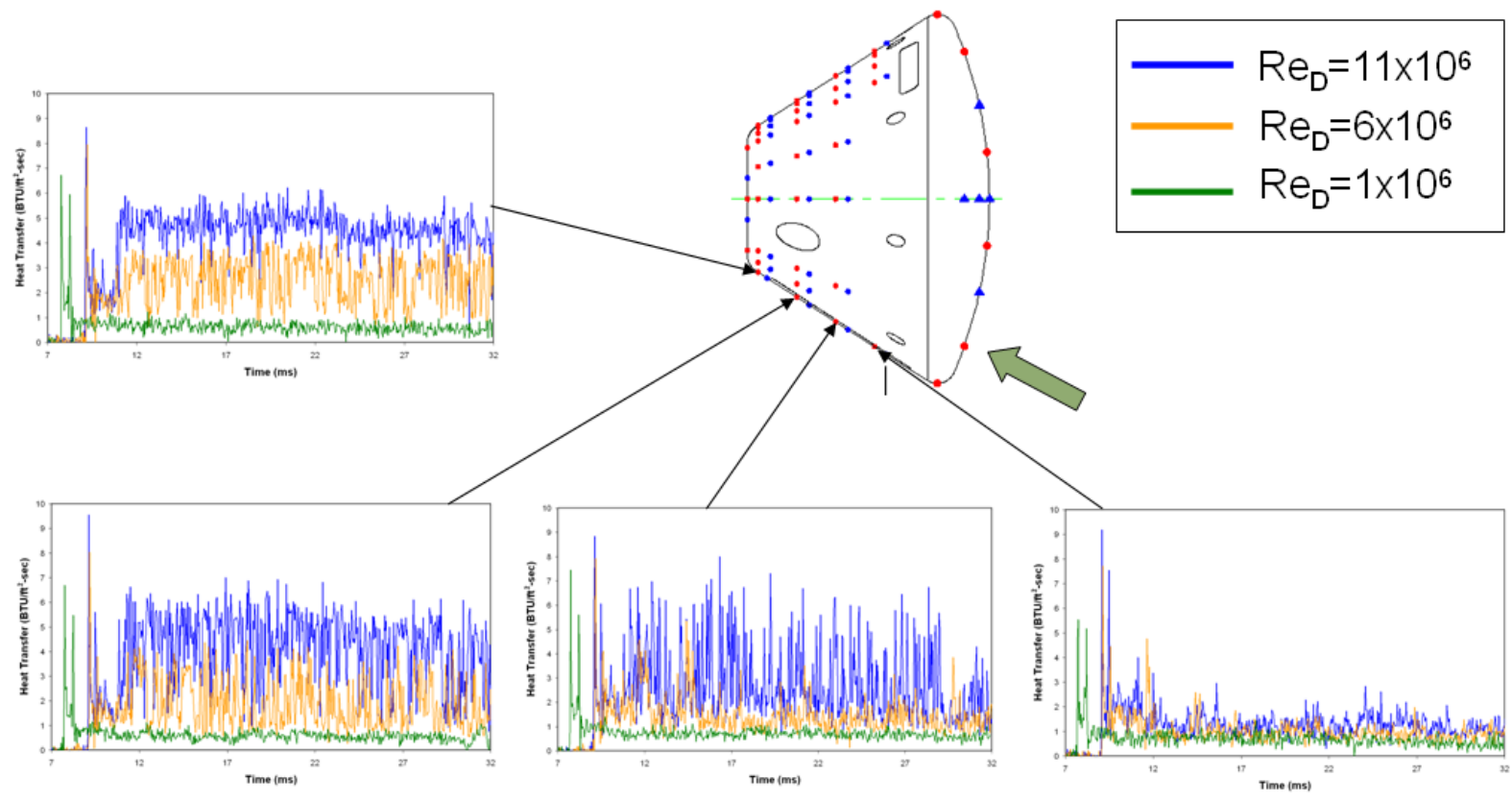

Figure 9. Heat Transfer Measurements on $180^{\circ}$ Ray for Three Reynolds Numbers Showing Transition to Turbulence 
signs of the same. This trend proceeds to the lower lefthand image when the highest condition is now more turbulent than laminar. In the final image in the upper left, the highest condition is beginning to show less fluctuating character indicating that it is at the end of the intermittent region and is fully turbulent. At this same point, the middle Reynolds number is transitional and the lowest Reynolds number has remained laminar for all traces.

For such a case, transition is easy to define in the sense that it is easy to pick out based on our previous experience on a variety of model shapes and flows. In the separated region, the issue is not as clear as Fig 10 indicates showing the time-history traces of the gages along the most leeward ray of sensors. Here again, the same three runs are shown at three different Reynolds number conditions as for the windward ray. The freestream flow moves from right to left, but the flow adjacent to the surface in this region is most likely moving in the opposite direction. Unlike before, here there is no progression with running length as there was on the attached ray. In fact, the traces farther aft (presumed to be upstream) tend to show more fluctuating character than the traces to the right. This may be more due to their proximity to the sting than any relation to streamline running length. However, we observe that with increasing Reynolds number there is a general, overall increase in fluctuating character for all traces. It is impossible to be sure that this is transition in the classic sense, but it does seem to suggest a greater tendency toward turbulence or at least flow instability.
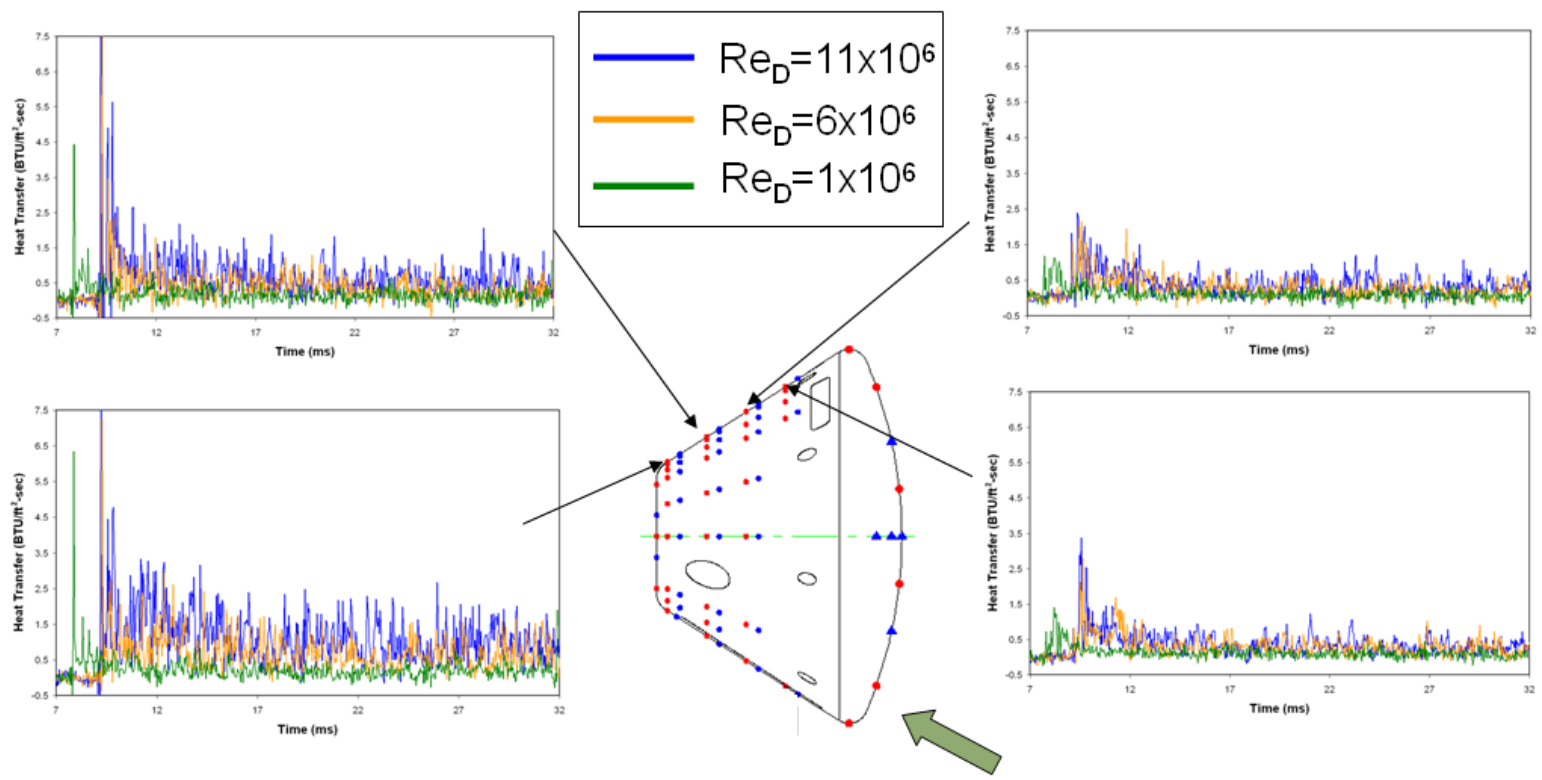

Figure 10. Heat Transfer Measurements on $0^{\circ}$ Ray for Three Reynolds Numbers Showing Fluctuating Character

\section{Measurements of Base Heating with Round Centered, Diamond Centered, and Split Stings}

Measurements were made using the sensors defined in Fig 6 for conditions given in Table 1. For all cases, the angle of attack of the spherical capsule was $28^{\circ}$ and the isothermal surface temperature was nominally $530^{\circ} \mathrm{R}$ $(293 \mathrm{~K})$. The runs were made at freestream Reynolds numbers ranging from nominally $1 \times 10^{6}$ per foot to $24 \times 10^{6}$ per foot, or $0.8-20 \times 10^{6}$ based on model major diameter. Enthalpy was varied somewhat in this study to maintain a minimum level of heating that provided sufficient signal-to-noise at very low Reynolds numbers. The large number of sensors employed in the study creates a large volume of data, both transient and time-averaged. A reduced set of data is presented here to highlight some of the major trends observed in the measurements. For this reason, several representative rays were selected from the dataset to most clearly communicate the results obtained. Time-averaged data is shown, where the time-averaging has been performed across 30 or more flow times at the last of the steadystate test time after the initial transient establishment is completed.

In all following images, the freestream flow is moving from right to left as indicated by arrows in each image, and the capsule is oriented such that the windward, $180^{\circ}$ ray is on the bottom and the leeward, $0^{\circ}$ ray is on the top. The heat transfer profiles are all plotted using the axial coordinate of each sensor so that each graph is oriented in the same direction as each capsule picture it is pointing to. In most plots, the Reynolds number based on 
Table 1. Listing of Freestream Conditions for Each Run Considered in Sting Comparison Study

\begin{tabular}{|c|c|c|c|c|c|c|c|}
\hline Run \# & Mach & $\begin{array}{c}\text { Velocity, } \\
\text { ft/s }\end{array}$ & Temperature, ${ }^{o} R$ & $\begin{array}{c}\text { Pressure, } \\
\text { psia }\end{array}$ & $\begin{array}{c}\text { Density, } \\
s l / f t^{3}\end{array}$ & $\operatorname{Re}_{D} \times 10^{6}$ & $\begin{array}{c}\text { Reference } \\
\text { Heating, } \\
\text { btu/ft }{ }^{2}-s\end{array}$ \\
\hline 2 & 6.41 & 3617 & 133 & 0.590 & $3.74 \times 10^{-4}$ & 10.8 & 12.90 \\
\hline 3 & 6.43 & 3631 & 133 & 0.349 & $2.18 \times 10^{-4}$ & 6.3 & 10.10 \\
\hline 4 & 6.46 & 4240 & 180 & 0.057 & $2.66 \times 10^{-5}$ & 0.7 & 6.96 \\
\hline 5 & 6.45 & 3262 & 106 & 0.823 & $6.49 \times 10^{-4}$ & 21.6 & 10.00 \\
\hline 8 & 6.39 & 3481 & 123 & 0.276 & $1.88 \times 10^{-4}$ & 5.6 & 7.55 \\
\hline 9 & 6.42 & 3507 & 124 & 0.601 & $4.06 \times 10^{-4}$ & 12.2 & 11.50 \\
\hline 10 & 6.45 & 3253 & 106 & 0.806 & $6.39 \times 10^{-4}$ & 21.3 & 9.67 \\
\hline 11 & 6.46 & 5424 & 295 & 0.729 & $2.08 \times 10^{-4}$ & 4.1 & 50.00 \\
\hline 12 & 6.39 & 3480 & 124 & 0.815 & $5.53 \times 10^{-4}$ & 16.6 & 13.00 \\
\hline 13 & 6.48 & 3198 & 101 & 0.398 & $3.30 \times 10^{-4}$ & 11.4 & 6.32 \\
\hline 14 & 6.35 & 4378 & 199 & 0.069 & $2.91 \times 10^{-5}$ & 0.7 & 8.32 \\
\hline 16 & 6.35 & 3502 & 127 & 0.289 & $1.92 \times 10^{-4}$ & 5.6 & 7.94 \\
\hline
\end{tabular}

diameter is given with the abbreviation " $M$ ” indicating millions or $\times 10^{6}$. Further, extensive effort was placed into non-dimensionalizing the data in a way that allows for different runs to be compared in the fairest way possible. Several schemes were considered including a Stanton number product with root and one-fifth power or Reynolds number, which we know to be useful in analyzing attached flow heating as they collapse laminar and turbulent heating profiles respectively. However, the best way to study the data in the wake region seems to be to nondimensionalize it using a Fay-Riddell heating value for a sphere matching the radius of curvature of the forebody. This value approximates the stagnation point heating of the laminar forebody, and for a completely laminar flow, we might anticipate that the base heating will scale as a percentage of this laminar forebody value.

The results obtained using the round, centered sting arrangement (Figs 4(a) and 5(a)) are summarized in Fig 11 for the $0^{\circ}, 90^{\circ}$, and $180^{\circ}$ rays of heat transfer. Several things may be noted, starting first with the $180^{\circ}$ ray pictured lower, left. Here, the transition process that was highlighted in Fig 9 is shown in a time-averaged sense, where the data shows three trends indicative of laminar, transitional, and turbulent attached flow. The reduction in slope near 3.0" for Run 2 suggests that at the last sensor the boundary layer is nearing a turbulent state. It is

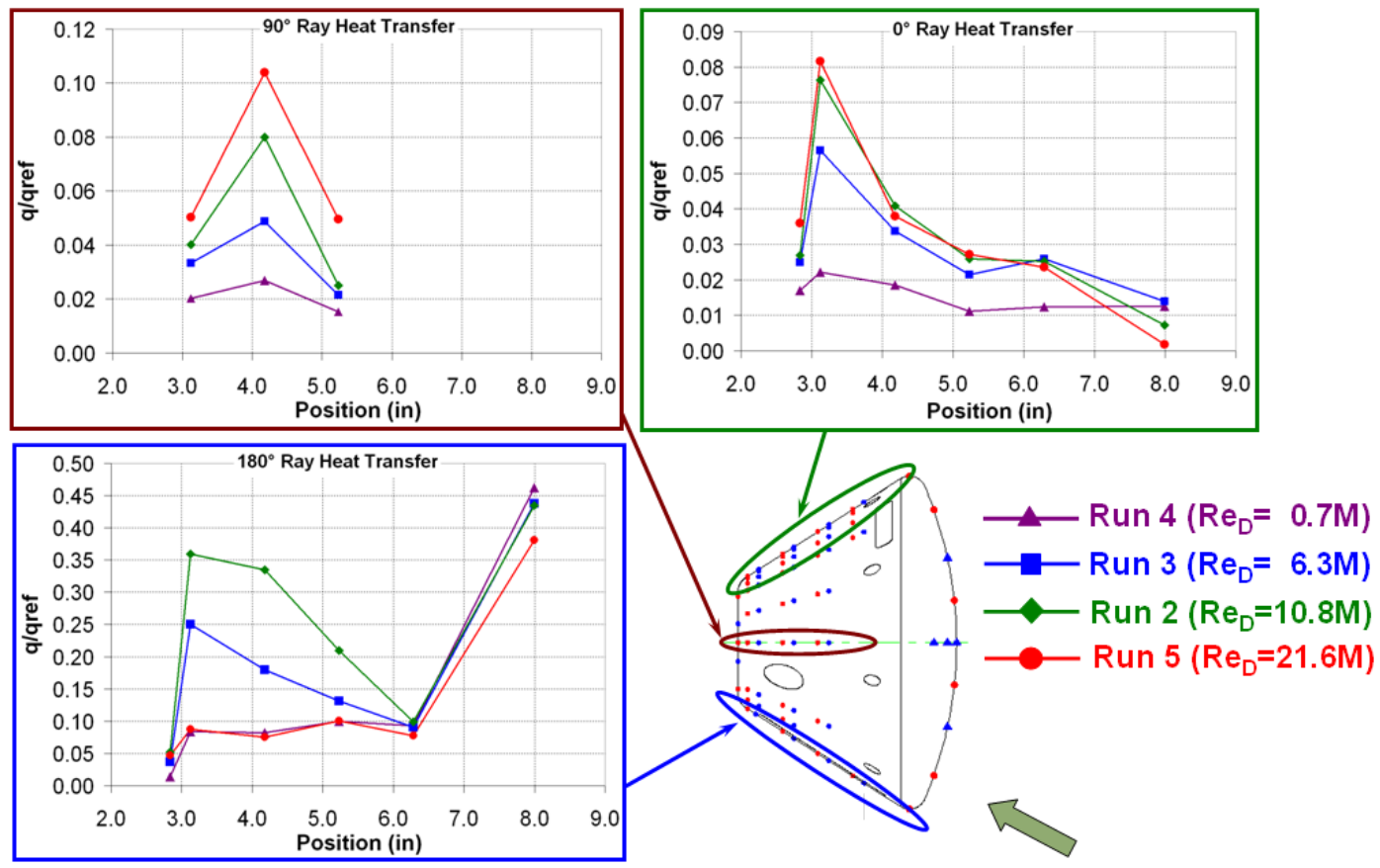

Figure 11. Heat Transfer Measurements on $0^{\circ}, 90^{\circ}$, and $180^{\circ}$ Rays for Round, Centered Sting Mount 
interesting to note that the highest Reynolds number case (Run 5) remains laminar on this attached ray, but it should be noted that Run 5 has a lower enthalpy than the others, suggesting possibly that there is some total enthalpy effect or wall to total temperature effect to the transition process on this shape. Since investigating the transition process for attached flows was not a primary objective of the experimental study, this phenomena was not considered extensively. On the other rays, the relative heating generally increases with increasing Reynolds number, where Run 5 displays the highest overall relative heat load as might be expected despite the fact that it remained laminar on the attached $180^{\circ}$ ray. On the $0^{\circ}$ ray nearest to the attachment point of the sting, it is also worth nothing that a large spike in heating was exhibited at high Reynolds numbers. The two highest Reynolds numbers collapse here, with the transitional and the laminar runs showing significantly lower levels of heating in this region.

A similar set of results is shown in Fig 12 for the diamond shaped, centered sting (Figs 4(b) and 5(b)). Here, the same three rays are shown in the same positions in the image $0^{\circ}$ (upper right), $90^{\circ}$ (upper left), and $180^{\circ}$ (lower left). The trends are generally the same as with the round sting, where the level relative to a laminar forebody increases with increasing Reynolds number. On the attached symmetry plane of the $180^{\circ}$ ray, the additional runs clearly captured the turbulent overshoot and decay process at higher unit Reynolds numbers and show a turbulent collapse at the most aft position for two high Reynolds number runs. Run 10 will be discussed further in the next section. A collapse was also obtained at least at the peak of the $90^{\circ}$ ray and again at the peak nearest the sting at the $0^{\mathrm{O}}$ ray. The peak level on the $0^{\mathrm{O}}$ ray is similar but slightly larger for the diamond sting than the round sting, but further direct comparisons will be made between the two in the next section. There is some limited evidence for an effect of flow total enthalpy in this dataset on the $0^{\circ}$ ray since Run 10 at the highest Reynolds number (but at a much lower enthalpy than for Runs 9 or 12) does show a reduced relative heat transfer rate in the peak region.

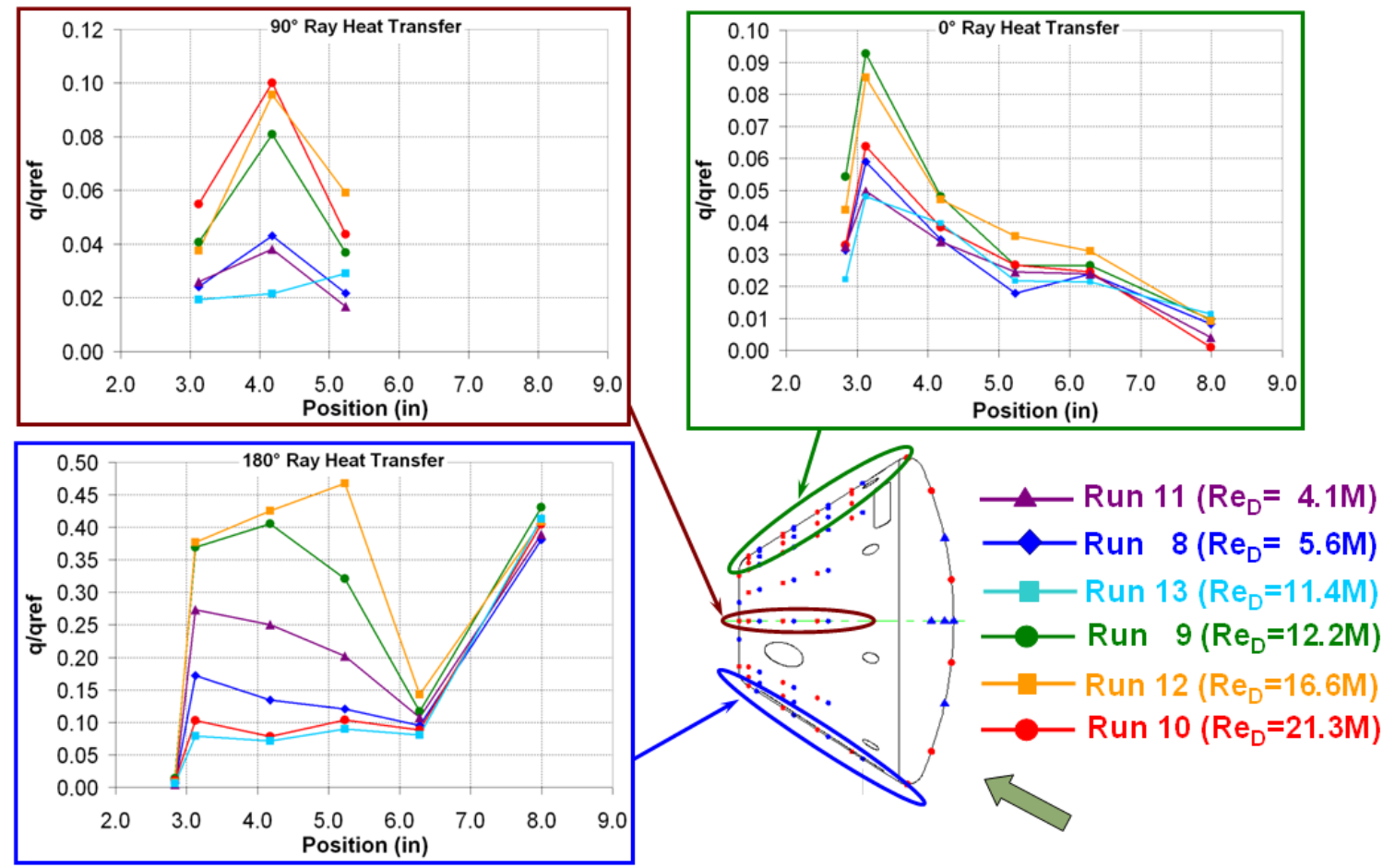

Figure 12. Heat Transfer Measurements on $0^{\circ}, 9^{\circ}$, and $180^{\circ}$ Rays for Diamond, Centered Sting Mount

Finally, results were obtained using the split sting arrangement (Figs 4(c) and 5(c)). These are shown in Fig 13. Because the split sting was designed to be minimally intrusive, as small as possible a diameter was selected for the rods which were mounted to the capsule. The stresses on the smaller rods were calculated to be considerably greater than for the two center mounted arrangements, so the maximum dynamic pressure in this series was limited. A catastrophic failure was not the primary concern for this self-imposed limit; the main issue was that large strains would cause significant deflections in the relatively flexible split sting mount and cause the angle of attack to change during the run and corrupt the test data. An imposed limit to the freestream dynamic pressure prevented the angle of attack from varying by any more than $0.1^{\circ}$ from the nominal $28^{\mathrm{O}}$. The runs selected that are shown in Fig 13 were 
picked primarily to make direct comparisons to the round and diamond stings that will be covered in more detail in the next section. In the case of the split sting results, the $135^{\circ}$ ray of heat transfer was selected for analysis rather than the $180^{\circ}$ ray as was shown in the previous studies because the $135^{\circ}$ ray is immediately in front of the point where the sting rod connects to the capsule body. In this region, both on the $135^{\circ}$ ray immediately in front of the sting and on the $90^{\circ}$ ray immediately behind the sting, there is significantly more activity with increasing Reynolds number than was observed in the center mounted configurations.
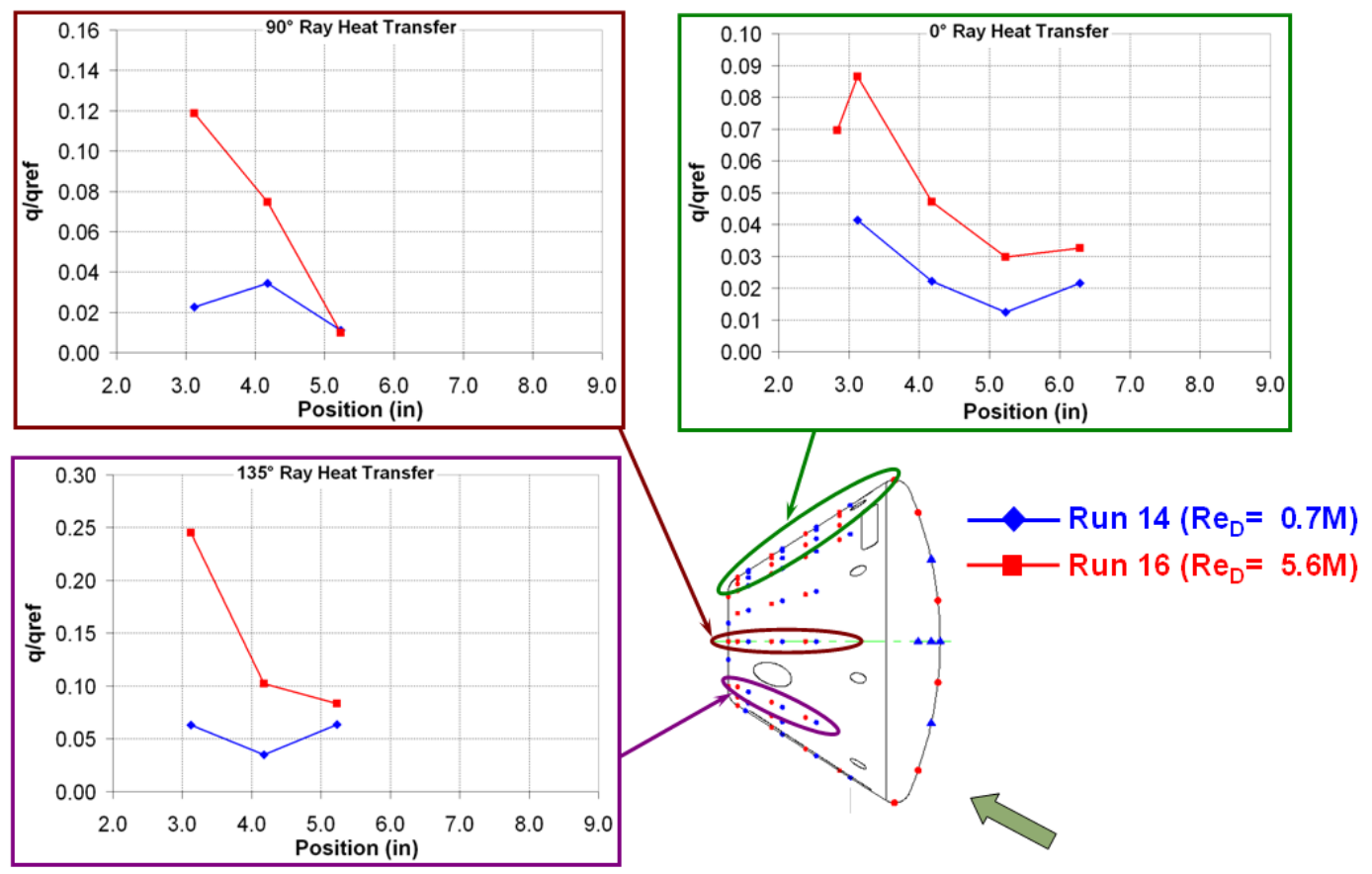

\section{Figure 13. Heat Transfer Measurements on $0^{\circ}, 9^{\circ}$, and $180^{\circ}$ Rays for Split Sting Mount}

Measurements have also been made at high frequency in an attempt to quantify the fluctuating character of the transitional and/or turbulent eddies in the separated wake region. At the outset of this effort, we had no prior experience or evidence to anticipate what the fluctuating unsteady character of the transitional or turbulent surface measurements would look like. The PCB pressure gages employed in this model are limited to about $20 \mathrm{kHz}$ frequency response, but the thin-film heat transfer gages should be capable of significantly higher frequency rates. Since they are a hand-painted platinum resistance element, they have very small thermal mass and should be capable of very high frequency response. We note, however, that this assumption has never been precisely measured to assess the limits of the capability of the gage in this regard. We know the limit to be high, but the threshold has not been quantitatively established. The electronics are also a major issue since they also have an upper limit in the frequency domain, so measurements have been made with the thin-films using three separate electronic amplification and acquisition systems with three different data rates. Typical power spectrum results are shown in Fig 14 for electronic systems limited to approximately $13 \mathrm{kHz}, 50 \mathrm{kHz}$, and $1 \mathrm{MHz}$ with correspondingly sufficient data acquisition rates to avoid Nyquist-Shannon aliasing to these limits. The results show generally broadband

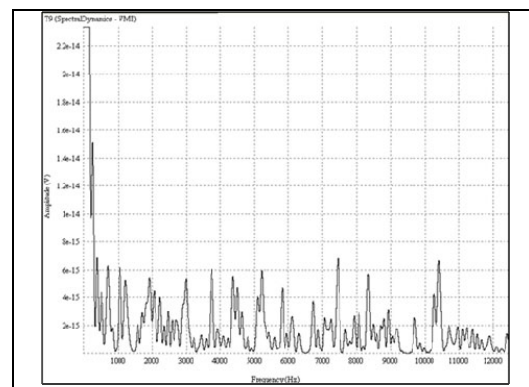

(a) $13 \mathrm{kHz}$ Resolution

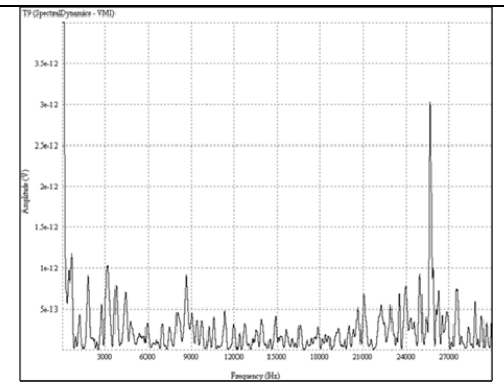

(b) $50 \mathrm{kHz}$ Resolution

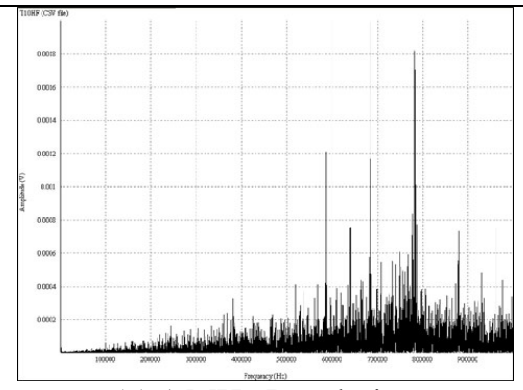

(c) $1 \mathrm{MHz}$ Resolution

Figure 14. Comparison of Power Spectrum of Heat Transfer Data Taken at Three Different Spectral Resolutions with Three Different Electronic Data Systems 
response in the lower frequencies with no clear dominant characteristic frequency. However, at significantly higher frequencies on the order of $500 \mathrm{kHz}$, there is dominant broadband activity with much greater overall power than was observed at the very low frequencies. Again, here in this frequency range, no single peak was detected, but there was generally significant noise over several hundred kilohertz span. We again stress that the response of the instruments at these frequencies have not been thoroughly characterized, but it does suggest some possible evidence of potential turbulence related activity.

\section{Comparisons on the Effect of Sting Mounting on Base Heating}

The most straight-forward analysis of the experimental data that can be made is to compare the measurements made with different stings in order to assess the differences in the influence of each one. Several conditions were selected where a similar run was made with multiple mounting arrangements. The comparison between the round (Figs 4(a) and 5(a)) and diamond centered (Figs 4(b) and 5(b)) stings is shown in Fig 15 for several Reynolds numbers. In each case, the round data set is shown with a solid trendline and the corresponding diamond dataset is shown in the dashed trendline with the same color code. In this way, like colors can be compared as being of equivalent freestream condition. As before, the data is non-dimensionalized using the laminar stagnation heating value listed in Table 1 which was based on our assessment of the best non-dimensional scheme to view the data.

As the data shows, there are only small differences between the round and diamond shapes overall. Most often, these differences are attributable to corresponding differences in the Reynolds number of the comparable runs. For example, the slightly more aggressive transition to turbulence on the $180^{\circ}$ ray when comparing Runs 2 and 9 (colored in green) is expected given that the diamond sting Run 9 was made at a Reynolds number of $12.2 \times 10^{6}$ while Run 2 was at $10.8 \times 10^{6}$. However, the points in the laminar and turbulent regions of this ray are very consistent. The same conclusion is generally true on most of the other rays such as the $90^{\circ}$ ray where a similar level and trend is evident between the two shapes. On the $0^{\mathrm{O}}$ ray, there are some non-trivial differences nearest the sting attachment points at the higher freestream Reynolds numbers. Run 9 is again slightly more elevated than Run 2 near the 3.0" location since the relative heating is higher by about $15 \%$, but this may again be attributable to the slight difference in freestream conditions. The difference in Run 5 and 10 is also about $20 \%$ at the peak location and those two runs have very similar freestream conditions. However, the difference is not dramatic enough to draw any definitive conclusion about differences between the stings.
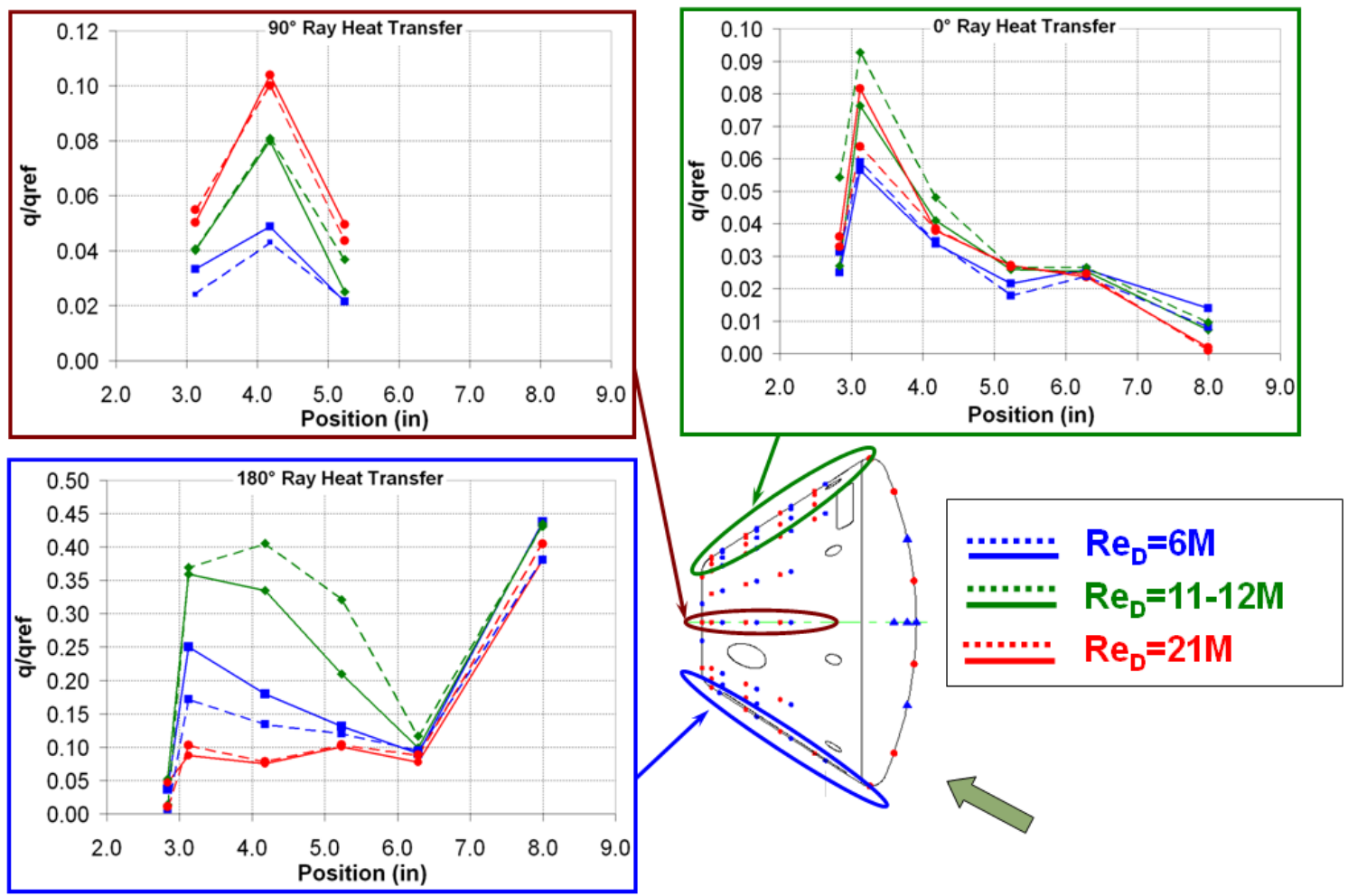

Figure 15. Comparison of Base Region Heat Transfer Measurements between Round Centered (solid lines) and Diamond Centered (dashed lines) Stings on $0^{\circ}, 90^{\circ}$, and $180^{\circ}$ Rays 
Next, the comparison is made between these two center mounted shapes, which we have just demonstrated to be similar overall, to the split sting. This comparison is shown in Fig 16 for two Reynolds numbers where the diamond sting is used for comparison at a Reynolds number of $0.7 \times 10^{6}$ and the round sting is used to compare at a Reynolds number of $5.6 \times 10^{6}$. In each case, the split sting result is shown with a solid trend line and the comparison run is given with a dashed line of the same color. Here the differences are much more significant so, while it is impossible based on surface measurements alone to definitively determine the causes for those differences, we may postulate several suppositions about possible explanations for the discrepancies. On the $135^{\circ}$ ray which is on the windward side of the aftbody, the comparison is quite similar at low Reynolds number, but a dramatic shift in behavior occurs at the higher Reynolds number. As the figure shows, these measurements are directly adjacent to the mounting point for the split sting member, so the increase in heating may be caused by shocks emanating from the sting mount at this position. A similar result is observed on the $90^{\circ}$ ray, which is located directly behind the split sting. Here again, the center mounted stings showed very little relative change in heating at this location for an increase in Reynolds number from 0.7 to $5.6 \times 10^{6}$, but the increase in heating is a factor of as much as six times on the split sting. A dramatic difference also occurs on the leeward centerplane or $0^{\circ}$ ray. Here, for both comparisons, the heating measured using the split sting is significantly higher than with the centered stings. At even the low Reynolds number of $0.7 \times 10^{6}$, the peak heating is higher by $100 \%$ and the increase at the higher Reynolds number is almost $50 \%$.

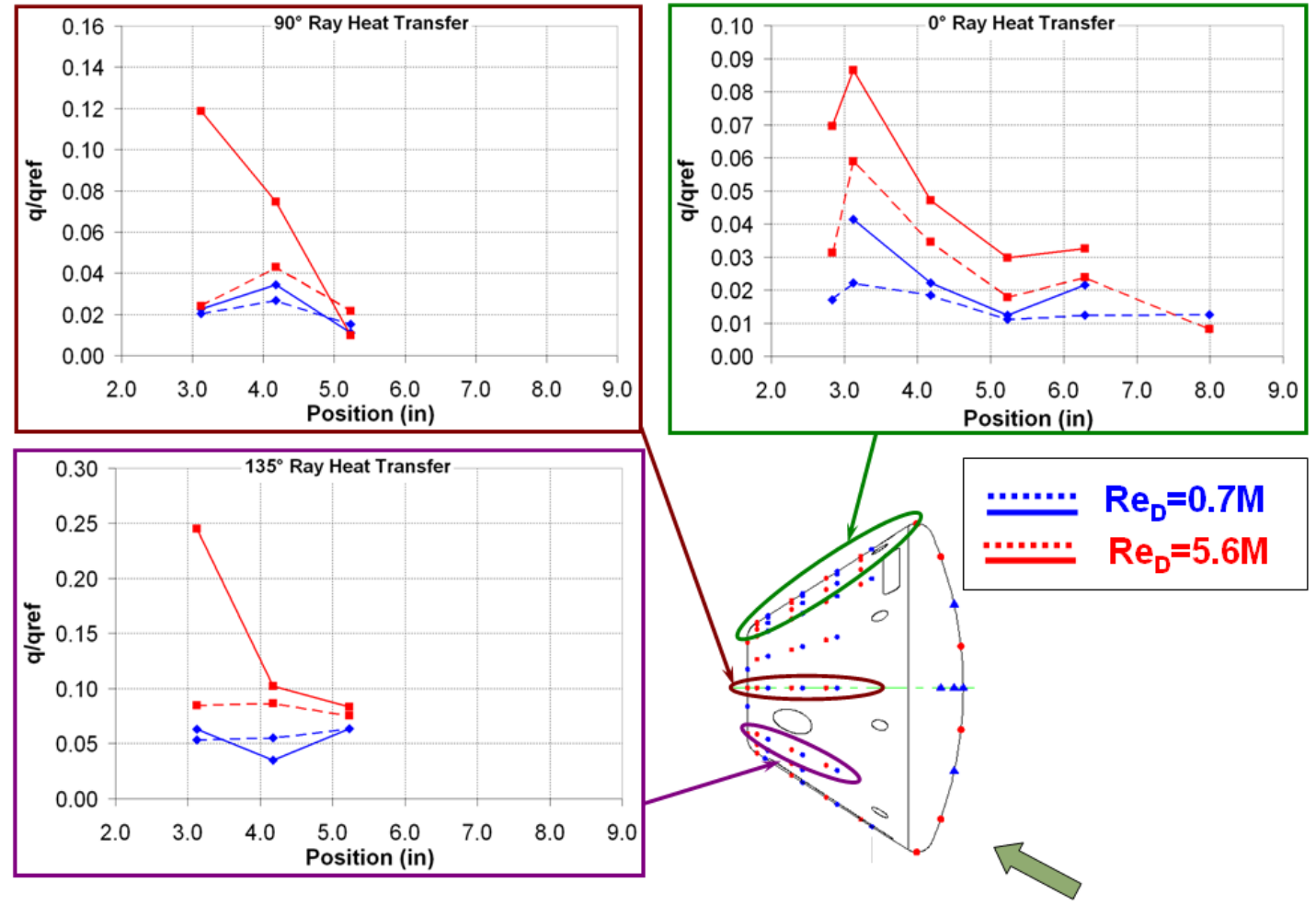

Figure 16. Comparison of Base Region Heat Transfer Measurements between Split (solid lines) and Centered (dashed lines) Stings on $0^{\circ}, 90^{\circ}$, and $135^{\circ}$ Rays

There are two possible explanations for this behavior on the $0^{\circ}$ ray. First, the split sting arrangement allows flow to freely pass on the centerplane. The higher heating observed on the split sting could be a result of higher momentum flow on the leeward part of the wake region that increases the relative heat transfer. Also, however, it is apparent that the peak relative heating of approximately $\mathrm{q} / \mathrm{q}_{\mathrm{REF}}=0.09$ observed at a Reynolds number of $5.6 \times 10^{6}$ is the same as the peak that was observed on the center mounted comparison in Fig 15 but at significantly higher Reynolds number. So it may also alternately be possible that the flow in this region has transitioned at much lower Reynolds numbers without the blockage caused by the center mounted stings. It is impossible to say for certain which explanation is correct from the heat transfer alone and supporting CFD simulations of these configurations to understand more about the flow patterns caused by the stings.

Next, the effect of Reynolds number is considered by analyzing several runs with the same total enthalpy but different freestream Reynolds numbers. This is shown in Fig 17, which plots cases ranging from $6 \times 10^{6}$ to $17 \times 10^{6}$ 


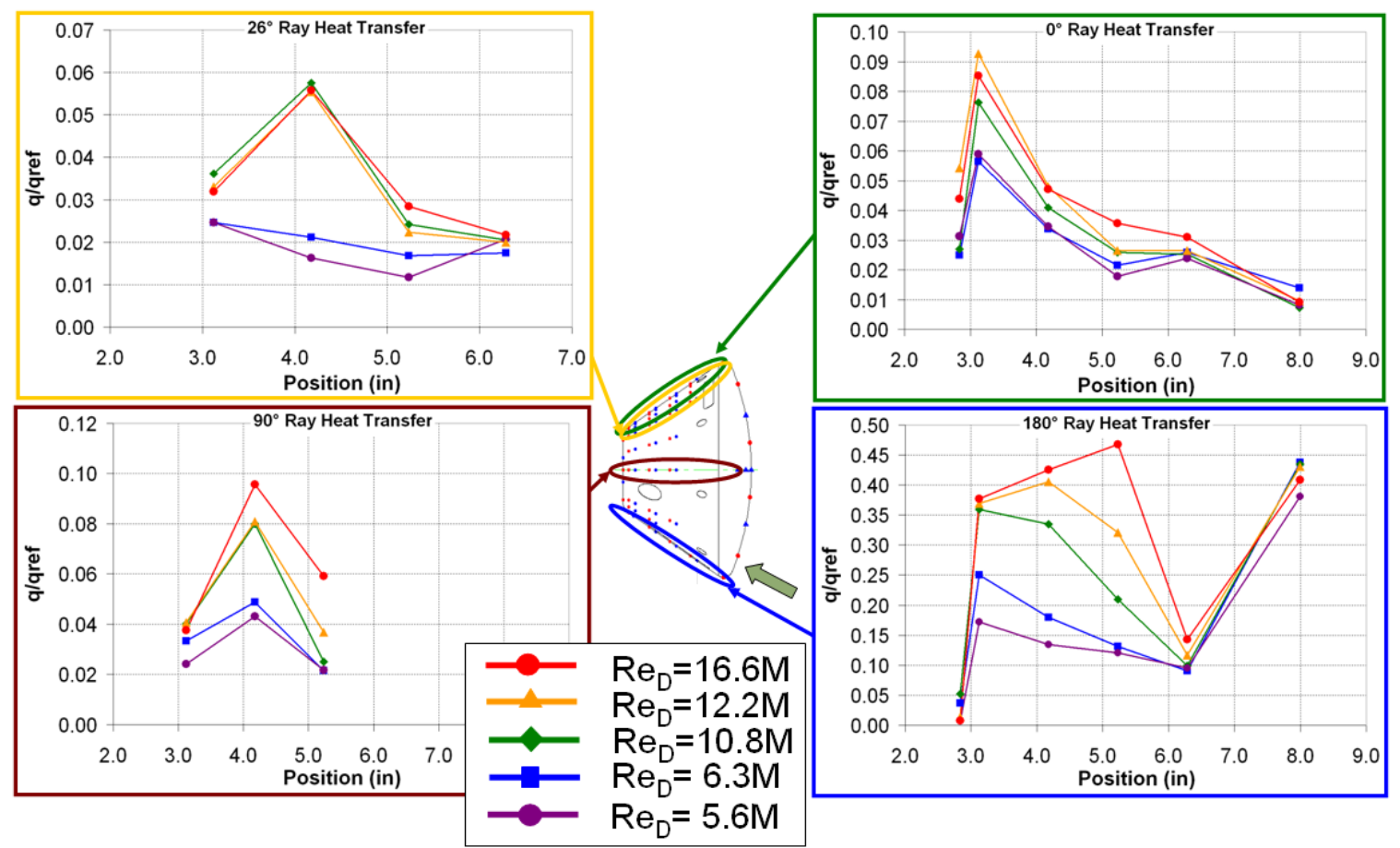

Figure 17. Comparison of Base Region Heat Transfer Measurements for Constant Total Enthalpy and Varying Freestream Reynolds Number on $0^{\circ}, 26^{\circ}, 90^{\circ}$, and $180^{\circ}$ Rays

based on diameter. As shown previously, the $180^{\circ}$ windward plane displays the classic transition, turbulent overshoot and decay to equilibrium turbulence level behavior. At higher Reynolds numbers, the process initializes more quickly and more of the process is captured upstream of the separation point at the back of the capsule. In the separated region of the wake, we observed generally higher heating levels at higher freestream Reynolds numbers. We again urge the reader to note that this conclusion is based on non-dimensional heating that is relative to a laminar stagnation point baseline. Therefore, this augmentation is in addition to the natural increase in laminar heating which results from a higher dynamic pressure in the freestream. Although it is less clear than for the attached flow, this enhancement may be thought of as increasingly turbulent behavior. It is also interesting to note that while the trend is to smoothly increase on the $0^{\mathrm{O}}$ ray, a different trend occurs just off the leeward centerline on the $26^{\circ}$ ray. Here, there is a sudden jump in average level and change in pattern near the aft part of the capsule. All of these measurements were made with either the round or the diamond center sting, and this observation occurs just on the outer edge of the downstream side of those stings. Therefore, it is reasonable to assume that this sudden change in character is due to a change in the shedding character of those stings, but analysis with CFD would be required to make a more specific conclusion.

Finally, the effect of flow total enthalpy is considered by looking at cases which vary the total enthalpy of the flow and hold the freestream Reynolds number constant. Two sets of comparisons are shown in Figs 18 and 19. In each comparison, runs are annotated with the enthalpy term $\Delta \mathrm{h}_{0}=\left(\mathrm{h}_{0}-\mathrm{h}_{\mathrm{W}}\right)$. Conditions are shown in blue for very low relative enthalpy $(0.2 \mathrm{MJ} / \mathrm{kg})$, green for medium relative enthalpy $(0.4 \mathrm{MJ} / \mathrm{kg})$, and red for the highest relative enthalpy $(1.2 \mathrm{MJ} / \mathrm{kg})$. In Fig 18, two cases at a low Reynolds number of $4 \times 10^{6}$ are shown. At this freestream Reynolds number, the attached windside $180^{\circ}$ ray shows earlier transition onset for a higher total enthalpy. This is consistent with the trend that we have observed on other attached flows such as cones, but this particular boundary layer seems very sensitive to small increases in flow total enthalpy. However, in the separated region there is a negligible impact at this Reynolds number as illustrated on the $0^{\mathrm{O}}$ ray. At a somewhat higher freestream Reynolds number of $13 \times 10^{6}$ in Fig 19, the effect of total enthalpy on the $180^{\circ}$ ray is the same, causing significantly earlier transition in the hotter flows. This comparison shows most clearly that there is a significant effect of enthalpy on the windside transition on the capsule body since the relative enthalpy here is only higher by a factor of two times. On the $0^{\mathrm{O}}$ ray, there is a more significant increase in the relative heat transfer than was observed at the lower enthalpy. Thus, we may conclude that the total enthalpy of the flow may cause augmented heating in the base region at a sufficiently high enough Reynolds number. It is unclear if this is related to transition occurring in the separated 


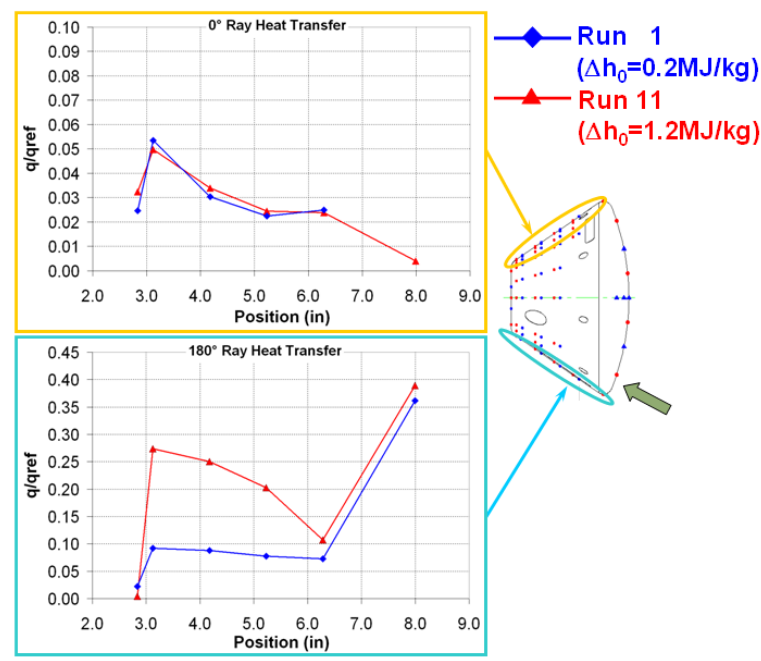

Figure 18. Comparison of Base Region Heat Transfer Measurements for Varying Total Enthalpy at a Reynolds Number of $4 \times 10^{6}$ on $0^{\circ}$ and $180^{\circ}$ Rays

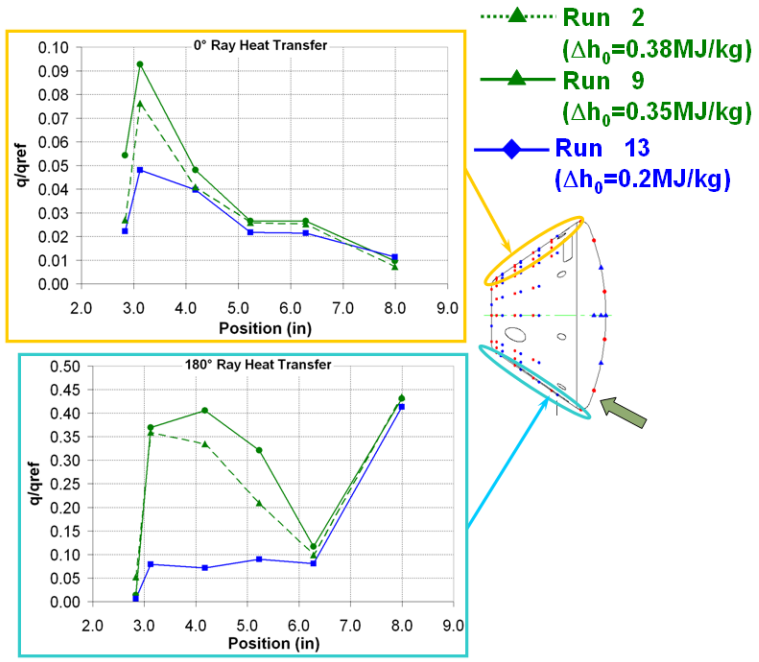

Figure 19. Comparison of Base Region Heat Transfer Measurements for Varying Total Enthalpy at a Reynolds Number of $11-12 \times 10^{6}$ on $0^{\circ}$ and $180^{\circ}$ Rays

flow, but it seems that there is some threshold for this phenomenon that is significantly different from the transition onset process in the attached boundary layer.

\section{Comparison of Experimental Data to Numerical Simulations}

Numerical fidelity is always tied to the design of the computational mesh. This is particularly true when using methods such as DES which are explicitly dependent on grid cell dimension. The topologies and sizing used in this work are developed from experience with the simulations presented by Barnhardt and Candler ${ }^{14}$. While not shown here, the sizing was further refined through a preliminary grid resolution study. To the extent possible, grid refinement was localized to the wake region of the leeside aftbody so that unnecessary refinement of the farfield was minimized. The final result is a fully three-dimensional mesh comprised of 9.7 million cells which includes a full $360^{\circ}$ of the capsule and sting without symmetry planes. The typical cell width in the wake is $2.5 \mathrm{~mm}$, or approximately $1 \%$ of the model diameter. Figure 20 depicts the surface and symmetry planes of the mesh. The mesh includes both the complete spherical capsule surfaces and the nearest sections of the round sting mount. It is approximately adapted to the bow shock of the forebody at angle of attack.
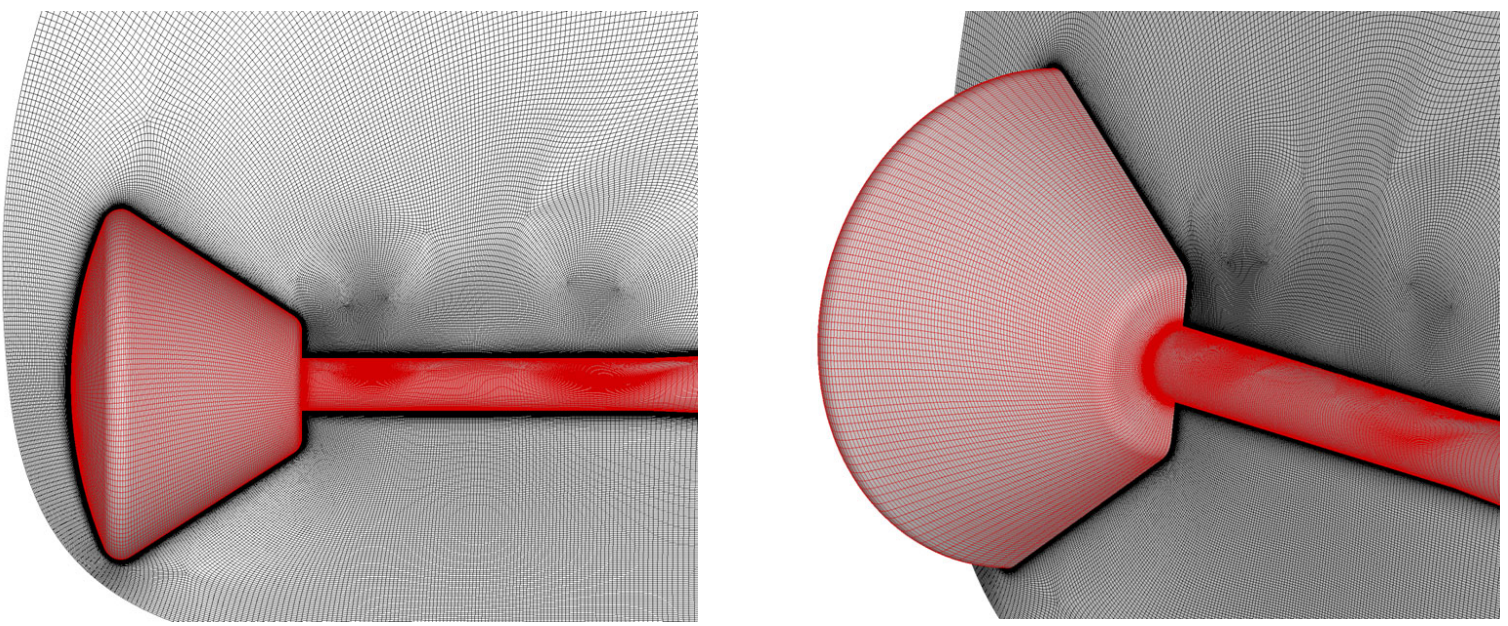

Figure 20. Centerplane and Surface Computational Mesh used in DES Study

Three of the cases listed in Table 1 were chosen for computational analysis in order to fully represent the transition from laminar to turbulent flows. Each case comes from the series of runs using the round centered sting. 
Based on the unit freestream Reynolds number and experimental data that was shown in Fig 11, test runs 4, 3, and 2 were deemed to be laminar, transitional, and turbulent, respectively. Flow visualizations of the temperature contours in the symmetry plane are shown in Fig 21.

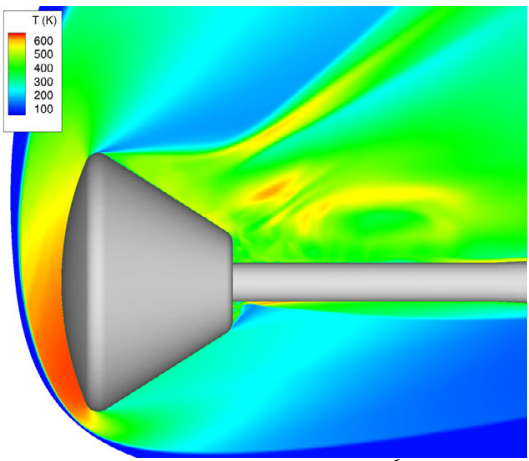

(a) Run $2\left(\operatorname{Re}_{\mathrm{D}}=10.8 \times 10^{6}\right)$

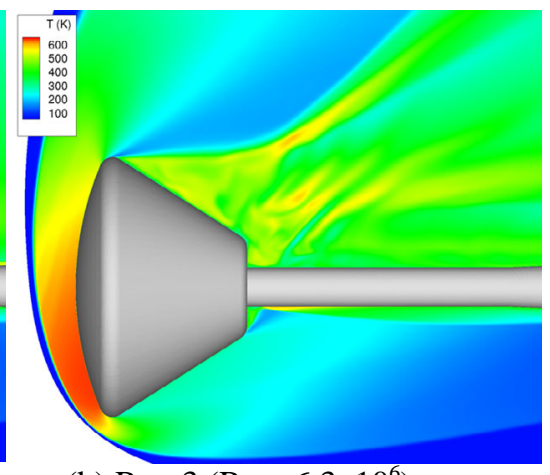

(b) Run $3\left(\operatorname{Re}_{\mathrm{D}}=6.3 \times 10^{6}\right)$

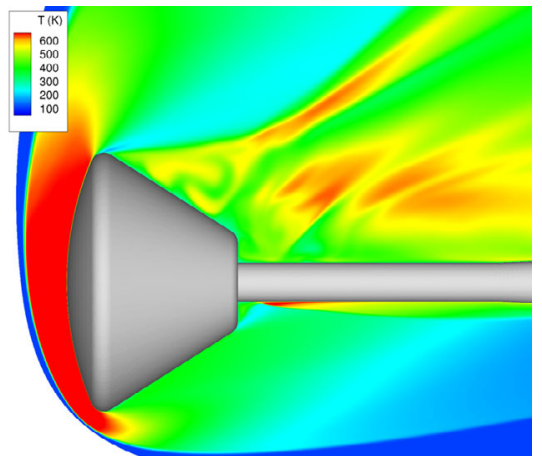

(c) Run $4\left(\operatorname{Re}_{\mathrm{D}}=0.7 \times 10^{6}\right)$

\section{Figure 21. Time-averaged Static Temperature Contours on the Centerplane}

Aside from the higher freestream enthalpy apparent from the forebody contours of Run 4, each of these runs shares essentially similar flow features. The flow along the windside aftbody is aligned with the freestream and therefore remains attached. The simulation predicts this region to be steady since there is no mechanism to model the intermittency of transition in this simulation. Depending on the freestream Reynolds number, the boundary layer in this region may transition to turbulence. The boundary layer then separates at the base of the capsule, creating a small recirculating region that interacts with the sting mount. As we move circumferentially about the capsule toward the leeside, the flow gradually begins to separate until we are in the wake region. At this point, the flow is fully separated and we see a marked increase in the unsteadiness predicted by the simulation. All cases are characterized by the shedding of vortices from the aftbody, although the range of apparent length scales is greater in the turbulent cases as expected. Furthermore, a secondary interaction exists between the larger separated capsule flow and that which emanates from behind the sting.

\section{A. Comparison to Laminar Wake (Run 4)}

We first examine the capabilities of the computational tools in the absence of turbulence as a means to highlight differences based on numerical rather than physical modeling. Wright, et al. $^{15}$ demonstrated a reasonable ability to predict laminar afterbody heating on the Apollo AS-202 command module using DPLR without regard for resolving the temporal evolution of the flow. Thus, at a minimum, we should be able to reproduce their accuracy levels given the heritage shared by DPLR and US3D.

Figure 22 depicts comparisons between experimental and numerical measurements of time-averaged heat transfer rate for Run 4 at all gage locations both on the forebody and aftbody. The pressure on the aftbody was also compared but found to be relatively constant over the entire separated region. In Figure 22, the gages are renumbered in a consecutive manner, ignoring the absent gages for ease of presentation. In this

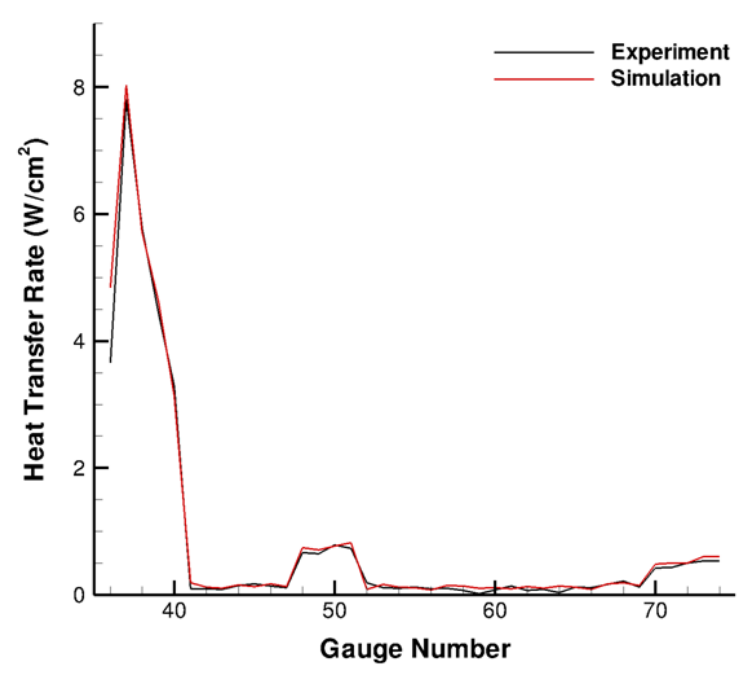

Figure 22. Comparison of CFD to Experimental Heat Transfer for all Gages for $\operatorname{Run} 4\left(\operatorname{Re}_{D}=0.7 \times 10^{6}\right)$ numbering scheme, the forebody instrumentation is represented by heat transfer gages [36 - 41] and the aftbody rays by the remaining gages. This type of plot provides a mechanism to compare all the data in a single plot for rapid evaluation of the validation. In the laminar flow, all of the remaining gages compare quite favorably, always within a few percent of the experimental value. This validates the basic numerical methodology and corroborates the findings of Wright et al. 


\section{B. Comparison to Transitional Wake (Run 3)}

We next turn our attention toward modeling of transitional and turbulent flows. For a transitional flow, we've simply selected Run 3 by virtue of it having the next highest Reynolds number, one which also coincides with previous experience simulating the transitional/turbulent flow of the Reentry-F vehicle. As noted earlier, turbulence modeling is handled by the Spalart-Allmaras equation with the standard DES modification.

Because the flow is transitional, we can anticipate that it will be difficult to accurately capture the experiment in the absence of some relatively sophisticated transition model. We therefore adopted the approach to simulate the experiment as both a laminar and fully turbulent flow with the hope that the results will bound the data. This is reflected in Fig 23, which shows results similar to that for Run 4 .

Pressure, as we would expect, is unaffected by the turbulence model. In both simulations, we obtain uniformly excellent agreement with the experiments so the pressure results have not been shown. Heat transfer, on the other hand, is considerably more erratic. First, we note the large differences between the experimentally measured forebody values and those predicted by the simulations. The laminar model here is clearly inappropriate, underpredicting the peak heating value by nearly $60 \%$. Still, one cannot say that the turbulent simulation performs perfectly either, particularly in the stagnation region between the nose and windside shoulder. This kind of behavior has been noted in several prior studies dealing with high Reynolds number forebody flows. An explanation for the disagreement is not currently available; however it is apparent in all of our simulations for which the forebody exhibited characteristics of turbulent behavior. Stagnation point augmentation has been explored by Holden ${ }^{20}$ for blunt body flows and some preliminary data presented by Wadhams, et al. ${ }^{18}$ seem to indicate an augmented level of heating in transitional flows that mitigates at higher Reynolds numbers. Away from the stagnation point, the discrepancy with the turbulent prediction can be explained by the turbulent overshoot heating of the transitioned boundary layer.

Otherwise, the aftbody simulations perform as expected. That is, the laminar solution uniformly underpredicts the experiment while the fully turbulent solution uniformly overpredicts it. This is best exemplified by the windside gages [47 -51] along the $\phi=180^{\circ}$ ray. Here we see that the laminar profile maintains an essentially flat character while the turbulent profile immediately peaks near the capsule shoulder (gage [51]) and then gradually decreases toward the base (gage [47]). In contrast, we see a slow ramping up of turbulent heating from the shoulder to the base in the experiment. By the time the flow reaches gage [47], the comparison between experiment and the fully turbulent simulation is actually quite good. This confirms that the flow is indeed transitional and that a more accurate simulation of this flow would be possible with the incorporation of a suitable transition model.

\section{Comparison to Turbulent Wake (Run 2)}

Let us now consider the fully turbulent case of Run 2. Because this case was expected to be fully turbulent, the simulation was performed using only the fully turbulent model. As with Runs 3 and 4, the results are presented in Fig 24 for the entire range of gages. Again, agreement of the pressure profiles is uniformly excellent. With regard to heat transfer rate, we see much improved agreement at nearly every gage, often within the measured standard deviation of the flowfield. Yet there are a couple of problematic regions. The difficulty of predicting stagnation point heating at high Reynolds number is once again evident. The windside boundary layer also has a slower transition relative to the computation. Nevertheless, these errors seem to be relatively localized and the aftbody gages are unaffected.

It is instructive to break down the presentation into the constituent rays of gages in order to gain some insight into the spatial dependence of our computational accuracy. This is shown in Fig 26, which depicts heat transfer comparisons for each ray of instrumentation. Here the computational results are denoted with a solid line and the experiment by symbols. The error bars reflect the measured standard deviation of the flow at each location and therefore provide an indication of the variation and unsteadiness of the local flowfield. We again note that this 
standard deviation is representative of physical fluctuations in the flow and is not necessarily representative of pointwise measurement error (the nominal uncertainty of thin-film gages is 5\%). As x-coordinate increases, we are moving from the shoulder toward the base; corresponding gage numbers are shown in red.

Principally, as is evident from Figs 25 and 26 as well, we see very good agreement for both pressure and heat transfer. For the most part, the computation lies within one standard deviation of the experiment, and when even that fails the disagreement is often within a few percent. There are however a couple of points which do stand out. First, with pressure we see significant disagreement at points numbered \#25 (64 ${ }^{\circ}$ ray), \#31 (135 ${ }^{\circ}$ ray), and \#35 ( $150^{\circ}$ ray). Each of these gages is spatially surrounded by other gages that perform well in the comparison, thus it is not immediately clear what is creating the discrepancy. One may note however that both points \#25 and \#31 lie near the junction of the conical afterbody and flat base. The inability to accurately predict these locations may well be due to the sharp flow separation and interaction between the aftbody flow and the flow behind the sting. In any case, the effect appears to be localized. Point \#35 has indications of being an outlier due to its rather benign location in the windside acreage and the fact that it is surrounded by three other gages which compare favorably with the experiment.

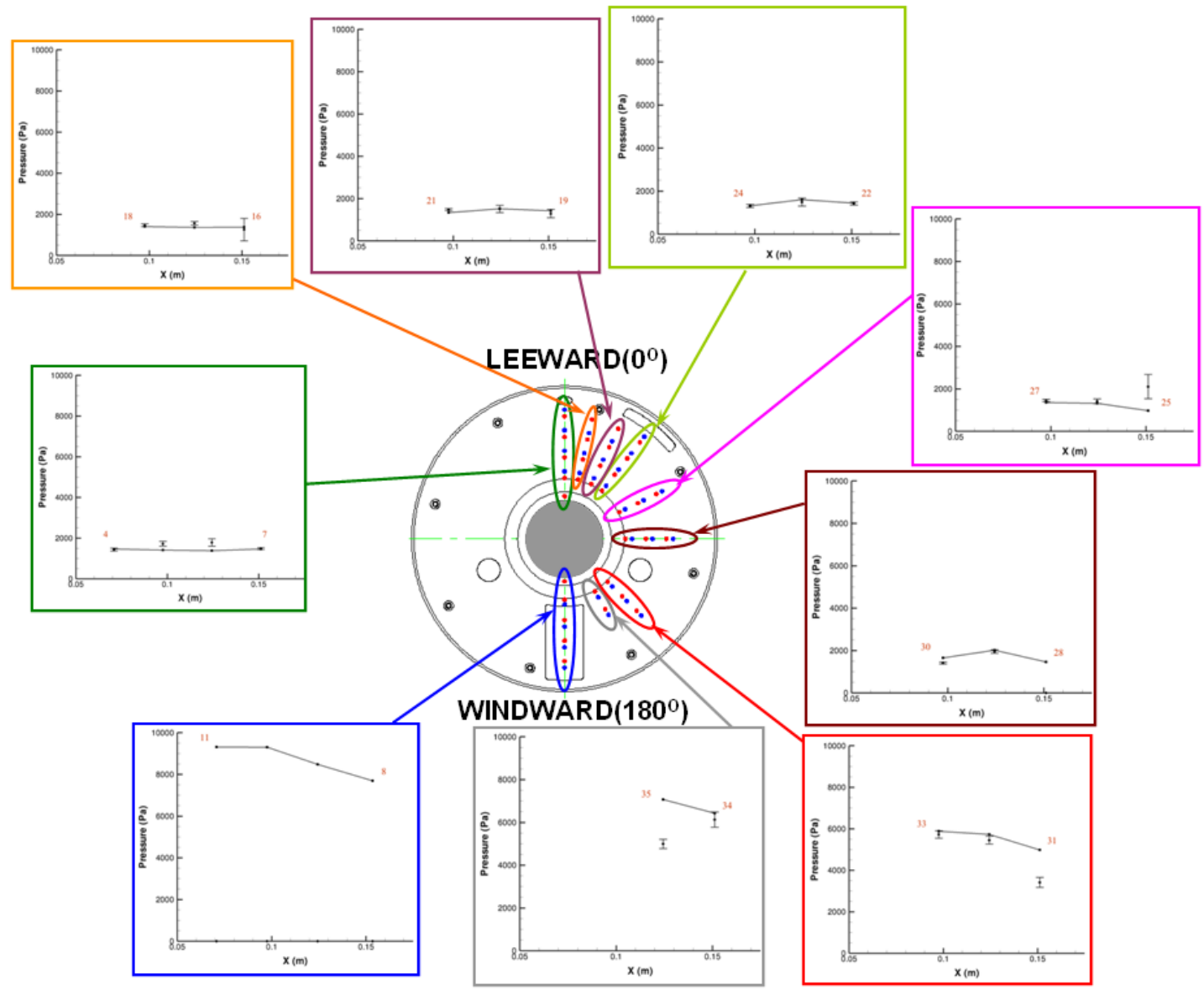

Figure 25. Comparison of CFD to Experimental Pressure for all Gages for $\operatorname{Run} 2\left(\operatorname{Re}_{D}=10.8 \times 10^{6}\right)$

American Institute of Aeronautics and Astronautics 


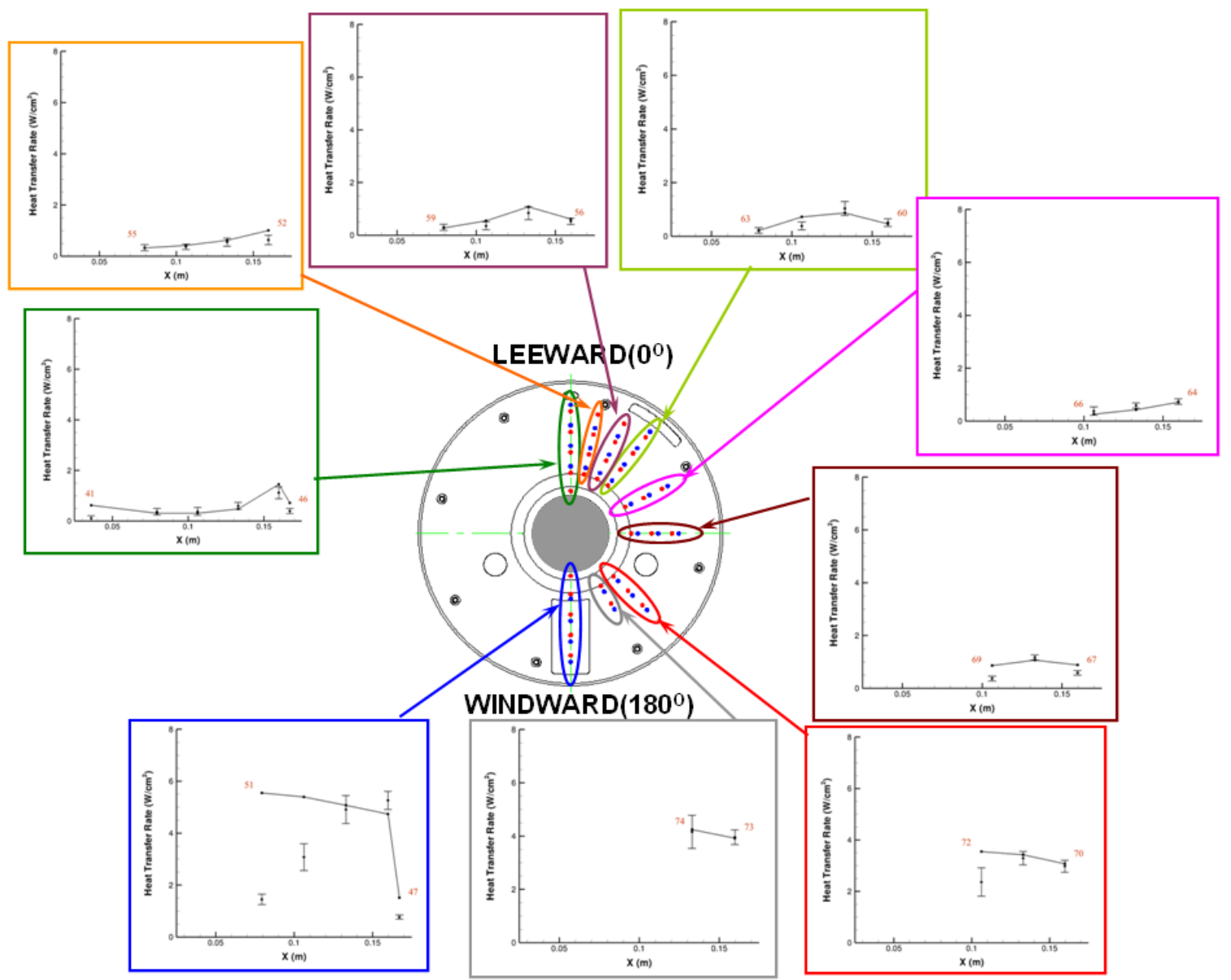

Figure 26. Comparison of CFD to Experimental Heat Transfer for all Gages for $\operatorname{Run} 2\left(\operatorname{Re}_{D}=10.8 \times 10^{6}\right)$

The most notable aspect of the heat transfer measurements is that the computation tends to overpredict results near separation points. This is particularly true for locations in which the flow is transitioning from the windside to the leeside, see for instance the $\phi=90^{\circ}$ ray. This seems to indicate an inability or lag in the computational model to accommodate a rapid expansion of the flowfield. Again, we note that this phenomenon is almost completely localized such that it has little or no impact on the gas subsequent to separation.

Finally, we may gain further insight into our numerical performance for this particular case by looking closer at the detailed transients of a few selected gages. While we cannot expect to perfectly replicate transient phenomena in our simulations, we can reasonably compare frequencies and amplitudes of oscillation. For this purpose, we have run our simulations over a time period comparable to the flow time achieved in the experiment and extracted transient data along the $\phi=0^{\circ}$ ray.

The resulting comparisons for gages on the $0^{\mathrm{O}}$ ray are shown in Fig 27. Note that increasing gage number indicates movement from the shoulder toward the base of the capsule. Gage 42 therefore reflects the state of the flow just subsequent to the strong expansion off the leeside shoulder. Here we see that the ability of the computation to correctly capture the amplitude and frequency of the flow is severely degraded. Interestingly, this does not detrimentally affect the time-averaged result. As we move away from the shoulder toward the base, we see a steady improvement in the simulation's prediction of oscillation frequencies and amplitudes. There is however a slight bias toward overprediction near the base, a fact which is apparent in the time-averaged results of Fig 26. These results suggest that local refinement of the computational mesh near the shoulder separation and wake/sting interaction may be necessary in order to accurately capture transient behavior in these regions. It is worth noting however that the discrepancies have a nearly negligible impact on the statistical results. 


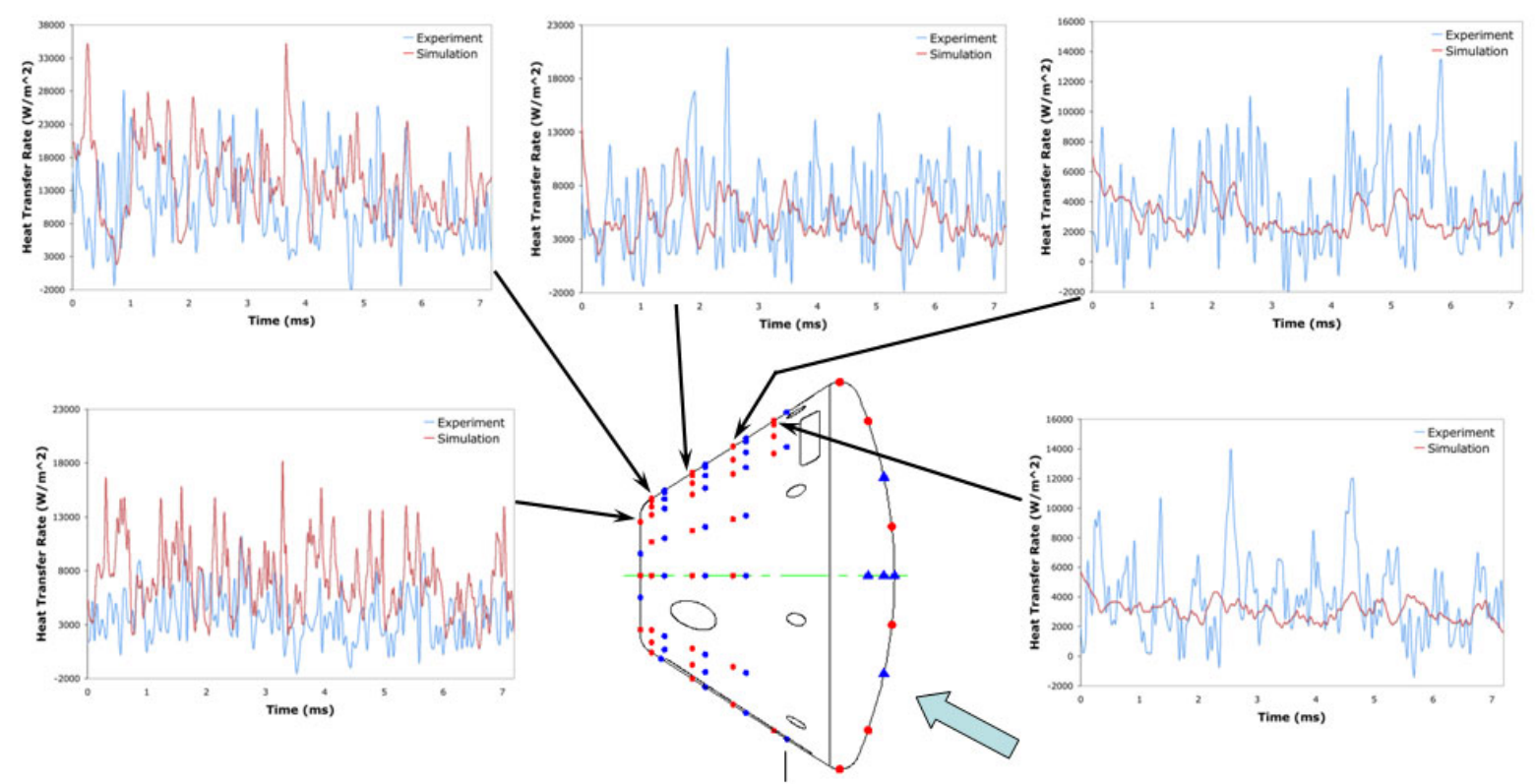

Figure 27. Comparison of CFD to Experimental Transient Heat Transfer for $0^{\mathrm{O}}$-Ray (leeward) for Run 2 $\left(\operatorname{Re}_{\mathrm{D}}=10.8 \times 10^{6}\right)$

\section{Experimental Study on the Effect of Jet Interaction in the Base Region of a Spherical Capsule}

An additional component to this program was to make measurements of the interaction occurring on the aftbody of the spherical capsule during an event where an RCS jet is firing. Control jets are typically employed to maintain attitude during reentry for vehicles such as Apollo or Orion, so characterizing the impact to the aftbody heat load from these jets is a critical issue for vehicle design ${ }^{21}$. The difficulty in this characterization is more complex when a cavity is considered in the smooth OML, representing a feature such as a recessed window well or something similar. An initial study was made both to provide a basic dataset of measurements for this problem and also to explore issues in making such measurements in the shock tunnel facilities. One primary concern, as for the smooth body measurements, was the length of time of and character of the transient start-up process so that future models could be properly sized and designed to make these critical measurements. To perform this study, the model was inverted by 180-degrees and the long insert piece shown in Fig 3 was replaced with an insert that contained a single cavity and a RCS jet oriented such that the gas discharged from the jet in the general direction of the cavity. The attitude of the model relative to the freestream was maintained at $28^{\mathrm{O}}$, but the cavity/jet system was placed on the leeward side of the body. Several thin-film heat transfer gages were installed on the surface of the insert and inside the cavity to monitor the heating changes with and without the jet interaction.

Several CAD renderings of this geometry are shown in Fig 28. The cavity was formed by cutting a rectangular section out of the curved surface of the aftbody, so the complex surface angles makes dimensioning the cavity difficult. The computed volume of the cavity was found using the CAD program to be $0.329 \mathrm{in}^{3}$. In addition, a contoured insert was built which reduced the size of the cavity as shown in Fig 28(c). This reduced size cavity was computed to have a volume of $0.146 \mathrm{in}^{3}$. The purpose of the smaller cavity was to measure the transient start-up

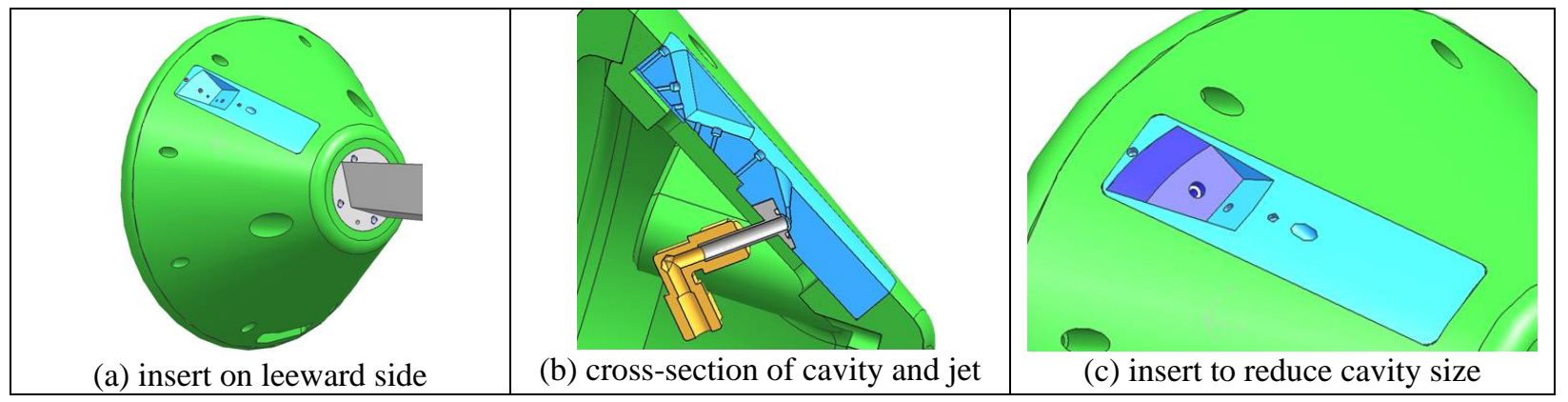

Figure 28. CAD Model of Cavity/RCS Jet Interaction Insert Installed in Spherical Capsule Model 
process on two different sized cavities to determine the effect of the cavity size on the time required. The RCS jet is a supersonic, conical jet with $10^{\mathrm{O}}$ half-angle and an exit to throat area ratio of 25:1. For nitrogen, the exit Mach number is about 4.0. The physical insert installed in the model is shown in Fig 29.

A Schlieren image of the jet firing on the aftbody is shown in Fig 30. The jet was operated using pure nitrogen at room total temperature at total pressures of 100psia and 200psia. Studies were made with freestream dynamic pressures ranging from 3 psia to 50 psia, and, of course, the local dynamic pressure of the flow in the wake will be very much lower than the freestream levels for any given case. Therefore, in all cases, the jet was at a significantly higher dynamic pressure than the surrounding flow to better represent conditions practical to flight.

Measurements for a gage on the surface of the insert and a gage at the bottom of the full-size cavity are shown in Fig 31 for a condition without the RCS jet on, which illustrates that the cavity takes somewhat longer to establish in the base region than a gage nearby on the surface. When the fully-established, time-averaged levels are compared, it should also be noted that the average level in the cavity is nominally 50\% higher than on the smooth surface nearby, indicating that both for temporal establishment requirements and for average heat load, the cavity is a limiting case and is critical to measure in the ground tests.

The effect of the jet was then considered on both the surface and the cavity heating. The effect of the jet on the cavity heating is shown in Fig 32. There are two points to consider in these two traces. First, the overall level of heating decreases somewhat because of the local cooling effect of the cold jet. This was a known issue at the outset of the experiment, but, since the goal of this particular study was to obtain data for code validation

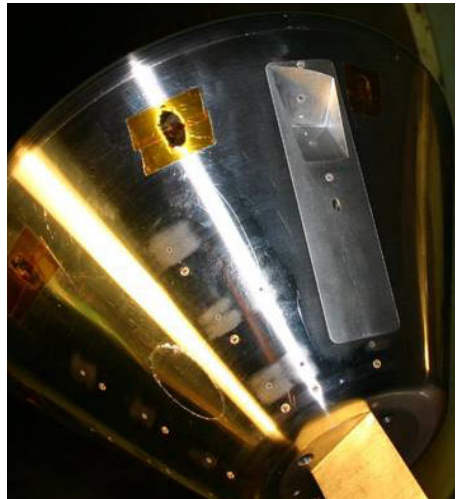

Figure 29. CAD Model of Cavity/RCS Jet Interaction Insert Installed in Spherical Capsule Model

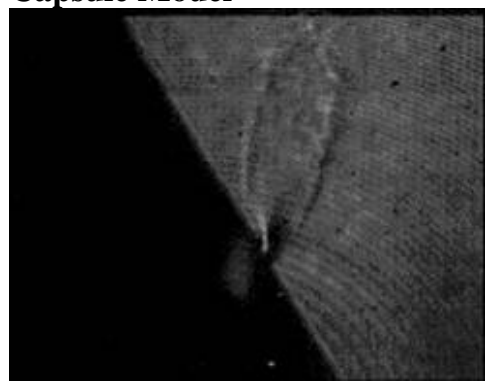

Figure 30. Schlieren Image of RCS Jet showing Barrel Shock

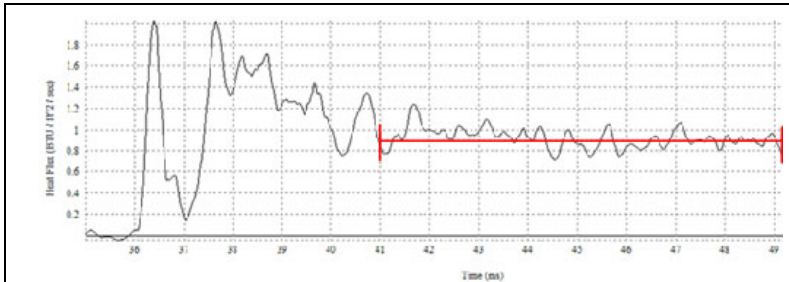

(a) Surface Gage

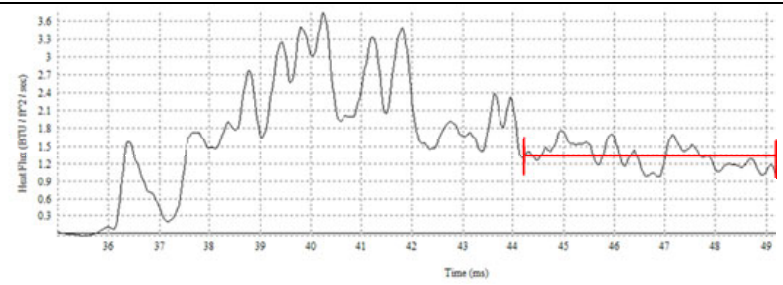

(b) Cavity gage

Figure 31. Typical Time History Measurements for Surface and Cavity Thin-film Heat Transfer Gage on Spherical Capsule Backshell without Jet Interaction Effect

and explore the unsteady transient interactions that occur in the jet/cavity region, a cold jet was sufficient to achieve both goals. For future studies related to the Orion flight where duplicating actual Orion reentry parameters is important, the jet will be heated to better simulate the hot chemical mixture of a real RCS exhaust. Second, and most important here, the start-up transient is significantly mitigated and the gage reaches the fully-established but fluctuating level much sooner (defined as the time period where any reasonably long average taken during the time interval produces the same average heating). As shown, the start-up peak in heating observed without the jet diminishes in both magnitude and duration when the jet is on. One possible explanation

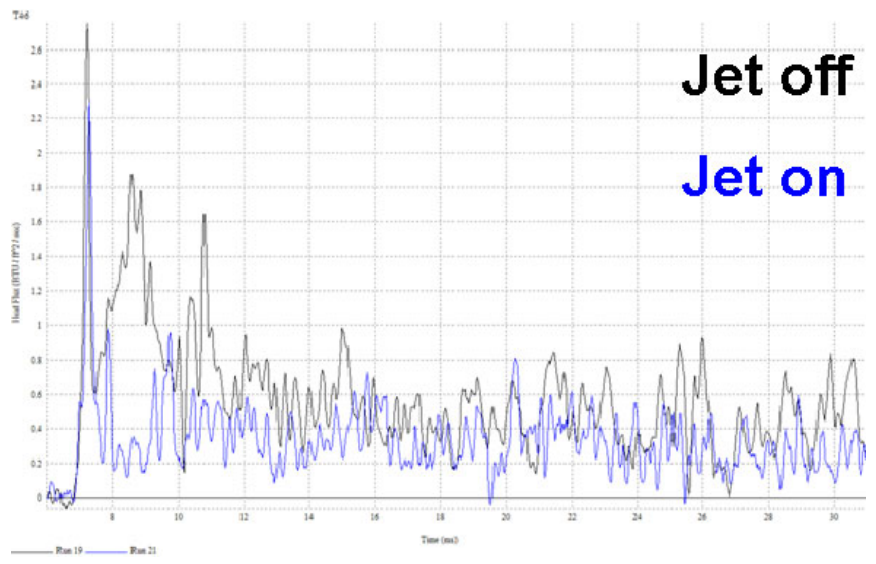

Figure 32. Typical Time History Measurements for Cavity Thin-film Heat Transfer Gage on Spherical Capsule Backshell with and without Jet Interaction Effect 
for this behavior is that the start-up transient occurs because a mass deficit exists in the wake and the time required for fully-established flow is at least partially driven by the time it takes to fill the base region with the required amount of gaseous mass. The injection of gas from the RCS jet fills in the base region much more quickly so that the flow reaches steady-state sooner than with no jet.

Finally, the results obtained in this study and from several other studies on spherical capsules of different sizes and under different conditions are summarized in non-dimensional form in Fig 33. The non-dimensionalization parameters chosen were the time required to reach steady-state heating, freestream velocity (which is a measure of total enthalpy) and the model diameter. This non-dimensional quantity is plotted versus freestream Reynolds number based on model diameter. The collapse achieved using this simple non-dimensionalization scheme with data on models of different physical scales and widely ranging freestream conditions is amazingly good. Several trends are shown - heating measured on the smooth body surface of the base region, heating measured at the bottom of a cavity in the smooth body surface with no RCS jets, heating measured on the smooth body surface with an RCS jet blowing onto the surface, and heating measured at the bottom of a cavity with an RCS jet blowing onto the cavity. The smooth body heating shows the smallest non-dimensional time requirement and the cavity-only flow shows the highest required non-dimensional time. Turning on the RCS jet requires more establishment time than for the smooth body, but it is interesting to note that the non-dimensional time required for the heating to the surface and in the cavity becomes about equal so that the lag in establishment observed on the cavity-only case is not present when the jet is included. For all cases considered, the condition with a cavity only and no RCS jet is the limiting case for model design. Also, the available data suggests that the non-dimensional time required for any of these situations decreases as the flow becomes increasingly turbulent and they asymptote to the same value at sufficiently high Reynolds numbers. Thus, we may conclude that a model must be designed for the lowest Reynolds number as the limiting case as the evidence shows that a less restrictive time is required at higher Reynolds numbers. This seems to be an effect of the increased turbulence in the flow, but it is interesting that the differences between the scenarios reduce to nothing in the limit at very high Reynolds numbers. More exploration of this conclusion is required with supporting numerical computations as there seems to be a physical mechanism causing this behavior that is important to understand these types of flows.

Capsule Backshell Time Establishment

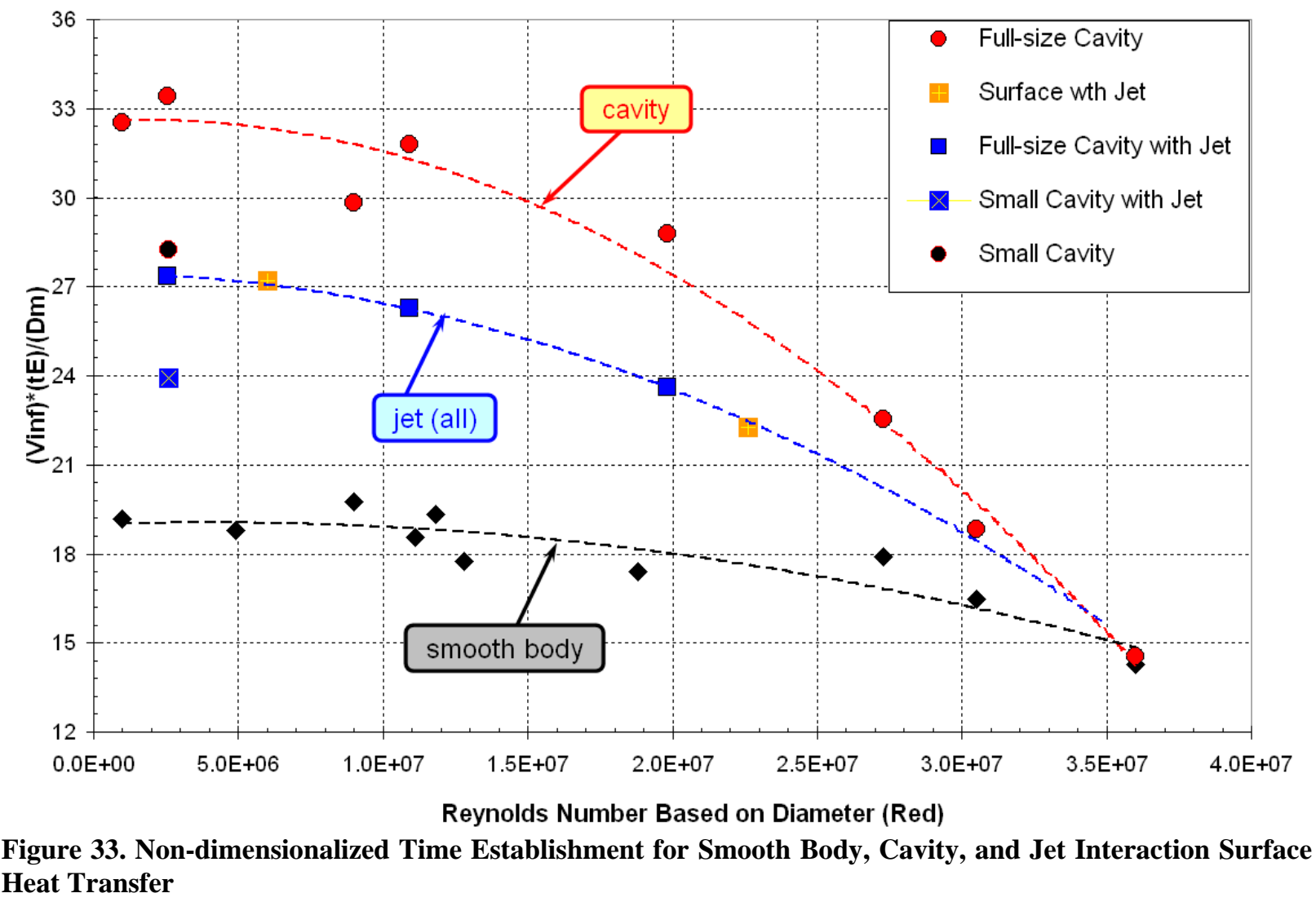


The effect of the size of the cavity is also apparent in the time establishment, where the smaller cavity takes considerably less non-dimensional time to establish than the larger one. This seems logical, but a more quantitative assessment can be made by comparing the time establishment requirement against the cavity size length scale as defined by the cubic root of the volume. An analysis was made assuming a linearly summing behavior where the time required for a cavity to stabilize is considered as the time required for the smooth body surface to stabilize (based on the model length scale), plus the time required for the cavity itself to stabilize (based on the cavity length dimension). By including the surface time establishment requirement as a cavity of zero volume as well as the measurements on the two different cavities at a fixed Reynolds number, Fig 34 shows that a remarkably linear behavior results. The number of available data points is very limited, but there is enough data to make an assessment of the time establishment required for the first-order design of future models. Therefore, we can conclude that the time required to establish the flow within the cavity itself can be computed as a summation of the time required for the smooth body surface as a function of freestream Reynolds number and velocity and the time for the cavity itself as a function of freestream velocity and cavity length scale.

\section{Experimental Study of Base Region Effects for Laminar Flows for Comparison to Navier- Stokes, DSMC, and Hybrid Code Validation}

Some data was also collected on a smaller spherical capsule body with a 5.75" major diameter that is presented for use in validating hybrid Navier-Stokes/DSMC codes. This smaller capsule is a scaled-down version of the split-sting mount studied in the previous section of this work. The data taken on the forebody of this smaller capsule has been studied extensively by MacLean, et $\mathrm{al}^{19}$. The detailed geometry and test conditions for the tests are given there so they will not be repeated here, but the model is shown installed in the facility in Fig 35. All tests were performed at a nominal Mach number near 10 at a range of enthalpies in both air and nitrogen from cold flow up to approximately $15 \mathrm{MJ} / \mathrm{kg}$. A range of conditions was considered in that work by comparing the measured forebody data to CFD simulations performed with the NASA DPLR code with specific emphasis on the surface catalytic response of the heat transfer gages and the thermochemical modeling of the freestream and shock layer chemistry. Here, some of the low density cases have been selected to use for DSMC applications since the wake region flow has very low densities that are borderline non-continuum.

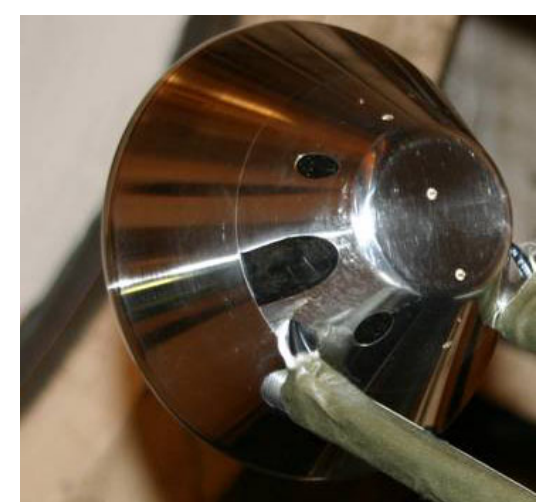

Figure 35. Small-scale Spherical Capsule Model with 5.75” Major Diameter Installed in Facility The Reynolds numbers based on diameter range from 35,000 to 52,000 in the freestream.

The surface pressure on the forebody and aftbody are shown in Fig 36 and the surface heat transfer is shown in Fig 37. Note that the pressure and heating scales on the graphics are log scale because the aftbody values are so much smaller than the forebody values. Trends for the runs are similar for all cases with only small variations in the data. Run 3 is shown in blue at a Reynolds number of 35,200, Run 14 is shown in red at a Reynolds number of 48,100, and Run 18 is shown in green at a Reynolds number of 52,000. 


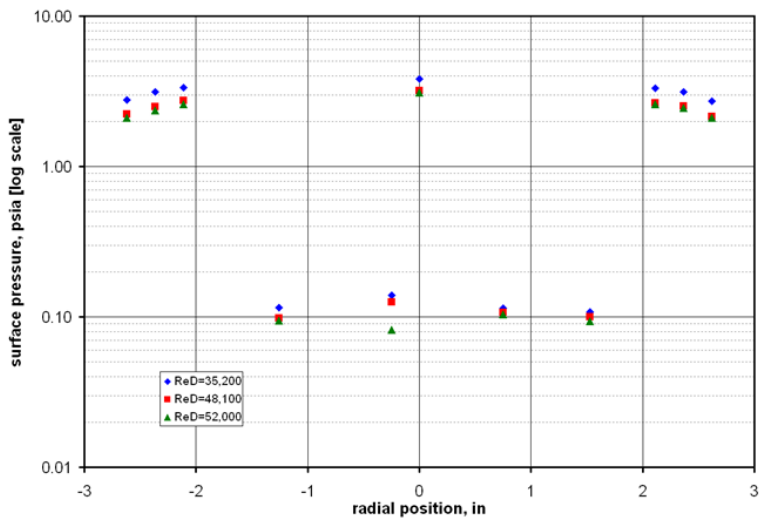

Figure 36. Small-scale Spherical Capsule Model with 5.75” Forebody and Aftbody Surface Pressure

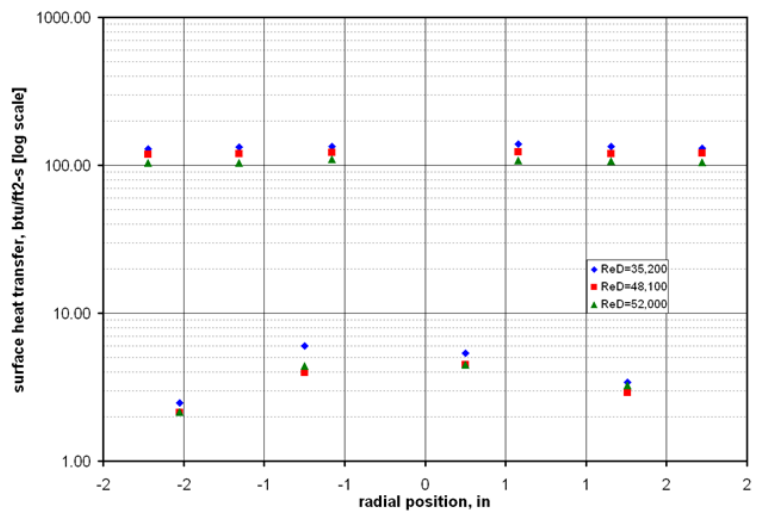

Figure 37. Small-scale Spherical Capsule Model with 5.75" Forebody and Aftbody Surface Heat Transfer

\section{Discussion and Conclusions}

Several series of experiments have been performed to study the aftbody heating of a spherical capsule vehicle to characterize the interactions with the separated base region flow and the sting attachment mechanism. The experiments have shown several trends within the data. First, the transition on the aftbody of the vehicle has been shown to be very dependent on the total enthalpy of the flow. On the attached side of the aftbody, the transition point moves steadily forward with increasing total enthalpy while on the leeward, separated side of the aftbody an increase in the relative level of heating was observed at sufficiently high Reynolds numbers. As the Reynolds number increases, an increasing level of relative heating in the leeward side of the aftbody was measured which may potentially be interpreted as transitional behavior.

While a change in shape of the sting produced only moderate changes in the overall character of heating to the aftbody, a change in location of the mount point of the sting produced a much more significant change in heating to the region. This was shown by comparing the center mounted sting geometries to the split mounted sting geometry under similar conditions. The higher level of heating observed on the split sting arrangement along the centerline may either be due to increased momentum along the centerline or due to enhanced transition by the unobstructed shear layer. Further significantly different heating patterns were observed immediately around the point at which the split sting supports mounted to the aftbody. Therefore, we can conclude that to properly characterize the complete aftbody of this type of vehicle, it is preferable to test with at least two different sting supports since each sting seems to disrupt the flow locally near it's attachment point. These configurations should ideally also be modeled with CFD to better understand the influence of the sting support.

An analysis on a limited number of runs with a high-quality, DES method developed within the US3D code has shown initial promise to model this type of flow across a range of Reynolds numbers and has several advantages over traditional RANS models. First, the DES method is inherently unsteady and it shows at least some capability to capture the unsteady fluctuations of the separated base flow. When properly time-averaged to compare with the experimental data, aftbody pressure and heat transfer were generally well captured to within one standard deviation of the measurements. Points which showed the largest discrepancies between the measurements and the CFD seemed to be tied to transition in the flow and in the region near the line of separation. No attempt was made to model transition with the CFD, so this limitation remains a central issue in simulating these types of flows.

Studies involving jet interaction were made to assess the influences of a combined RCS jet and cavity in the aftbody. Compared to the smooth body heating, it was found that the transient start-up of the aftbody heating took significantly longer with either a cavity or a jet; however, the effect of mass addition by the jet in the wake region lowered the transient establishment time requirement significantly over the cavity-only case. It was shown that the heating within the cavity is significantly higher than the surrounding surface heating and that the jet can cause a local cooling effect if room temperature gas is used instead of the hot exhaust gas that exits from the RCS in flight. Further, a simple non-dimensional scheme was found to design future models of detailed aftbody heating for running in short-duration facilities that can duplicate the true enthalpy of reentry flight.

Finally, a limited set of data is provided at very low Reynolds number for the purpose of validating hybrid Navier-Stokes/DSMC for aftbody separated flows. 


\section{Acknowledgements}

This work was completed with support from the NASA Aeronautics Research Mission Directorate under the Fundamental Aeronautics Program (FAP) through contract \#NNL07AA30C and grant \#NNX07AC28A.

\section{References}

${ }^{1}$ Lu, F.K. and Marren, D.E. Eds. Advanced Hypersonic Test Facilities. AIAA Progress in Astronautics and Aeronautics Series: Vol 198. Chapter 4. Reston, VA: American Institute of Aeronautics and Astronautics, 2002.

${ }^{2}$ Holden, M.S.; Wadhams, T.P.; and Candler, G.V. "Experimental Studies in the LENS Shock Tunnel and Expansion Tunnel to Examine Real-Gas Effects in Hypervelocity Flows”. AIAA Paper 2004-0916. January 2004.

${ }^{3}$ Parker, R.; Wakeman, T.; Holden, M.; and MacLean, M. "Measuring NO Freestream Concentration Using Quantum Cascade Lasers at CUBRC”. AIAA Paper 2006-0926. $44^{\mathrm{TH}}$ Aerospace Sciences Meeting \& Exhibit. Reno, NV: 9-12 January 2006.

${ }^{4}$ MacLean, M.; Candler, G.; and Holden, M. "Numerical Evaluation of Flow Conditions in the LENS Reflected ShockTunnel Facilities”. AIAA Paper 2005-0903. 43 ${ }^{\mathrm{RD}}$ Aerospace Sciences Meeting \& Exhibit. Reno, NV: 10-14 January 2005.

${ }^{5}$ Spencer, David; Blanchard, Robert; Thurman, Sam; Braun, Robert; Peng, Chia-Yen; and Kallemeyn, Pieter. "Mars Pathfinder Atmospheric Entry reconstruction”. AAS 98-146. AAS/AIAA Space Flight Mechanics Meeting, Monteray, CA. 9-11 February 1998.

${ }^{6}$ Wright, M.J.; Bose, D.; and Candler, G.V. “A Data Parallel Line Relaxation Method for the Navier-Stokes Equations”. AIAA Journal. Vol 36, no 9. Pgs 1603 - 1609. Sept 1998.

${ }^{7}$ MacCormack, R.W. and Candler, G.V. "The Solution of the Navier-Stokes Equations Using Gauss-Seidel Line Relaxation”. Computers and Fluids. Vol 17, No 1. Pgs 135 - 150. 1989.

${ }^{8}$ Candler, G.V. “Chemistry of External Flows”. Aerothermochemistry for Hypersonic Technology: Von Karman Institute for Fluid Dynamics Lecture Series. VKI LS 1995-04.

${ }^{9}$ Nompelis, I., T. Drayna, and G.V. Candler, "Development of a Hybrid Unstructured Implicit Solver for the Simulation of Reacting Flows Over Complex Geometries,” AIAA Paper 2004-2227. $34^{\mathrm{TH}}$ AIAA Fluid Dynamics Conference, Portland, 28 June 28 - 1 July, 2004.

${ }^{10}$ Nompelis, I., T. Drayna, and G.V. Candler, “A Parallel Unstructured Implicit Solver for Hypersonic Reacting Flow Simulation,” AIAA Paper 2005-4867, $17^{\mathrm{TH}}$ AIAA Computational Fluid Dynamics Conference, Toronto, Canada 6-9 June 2005.

${ }^{11}$ Spalart, P.R. and Allmaras S.R. “A One-Equation Turbulence Model for Aerodynamic Flows”. AIAA Paper 92-0439. 30 ${ }^{\mathrm{TH}}$ Aerospace Sciences Meeting \& Exhibit. Reno, NV: 6-9 Jan, 1992.

${ }^{12}$ Catris, S., and B. Aupoix, "Density Corrections for Turbulence Models," Aerospace Science and Technology, Vol.4, pp. 111, 2000.

${ }^{13}$ Spalart, P.R., W-H. Jou, M. Strelets, and S.R. Allmaras, “Comments on the Feasibility of LES for Wings and on a Hybrid RANS/LES Approach,” Advances in DNS/LES, 1st AFOSR International Conference on DNS/LES, 1997.

${ }^{14}$ Barnhardt, M. and G.V. Candler, "Detached Eddy Simulation of the Reentry-F Flight Experiment,”AIAA 2008-0625, 2008.

${ }^{15}$ Wright, M.J., D.K. Prabhu, and E.R. Martinez, "Analysis of Afterbody Heating Rates on the Apollo Command Modules, Part 1: AS-202," AIAA 2004-2456, 2004.

${ }^{16}$ Wadhams, T.; MacLean, M.; Holden, M.; and Mundy, E. "Pre-flight Ground testing of the Full-scale FRESH FX-1 at Fully Duplicated Flight Conditions - Part II,” AIAA Paper 2008-0639. 46 ${ }^{\mathrm{TH}}$ AIAA Aerospace Sciences Meeting \& Exhibit. Reno, NV: 7 - 10 January 2008.

${ }^{17}$ MacLean, M.; Mundy, E.; Wadhams, T.; Holden, M.; Johnson, H.; and Candler, G. "Comparisons of Transition Prediction using PSE-Chem to Measurements for a Shock Tunnel Environment.” AIAA Paper 2007-4490. 37 ${ }^{\text {TH }}$ AIAA Fluid Dynamics Conference \& Exhibit. Miami, FL: 25 - 28 June 2007.

${ }^{18}$ Wadhams, T.; et al. "Experimental Studies of the Aerothermal Characteristics of the Project Orion CEV Heat Shield in High Speed Transitional and Turbulent Flows,” AIAA Paper 2009-0677. 47 ${ }^{\mathrm{TH}}$ AIAA Aerospace Sciences Meeting \& Exhibit, Orlando, FL: 4 - 8 Jan 2009.

${ }^{19}$ MacLean, M.; Mundy, E.; Wadhams, T.; Holden, M.; Parker, R. “Analysis and Ground Test of Aerothermal Effects on Spherical Capsule Geometries.” AIAA Paper 2008-4273. 38 ${ }^{\mathrm{TH}}$ AIAA Fluid Dynamics Conference \& Exhibit, Seattle, WA: 22 26 June 2008.

${ }^{20}$ Holden, M.S.; Duryea, G.R.; Gustafson, G.Q.; Hudack, L.T. “An Experimental Study of Particle-Induced Convective Heating Augmentation,” AIAA Paper 1976-320, AIAA Fluid \& Plasmadynamics Conference, San Diego, CA: 14 - 16 July 1976.

${ }^{21}$ Buck, G.; Watkins, A.; Danehy, P.; Inman, J.; Alderfer, D.; Dyankonov, A. "Experimental Measurement of RCS Jet Interaction Effects on a Capsule Entry Vehicle,” AIAA 2008-1229. 46 ${ }^{\mathrm{TH}}$ AIAA Aerospace Sciences Meeting \& Exhibit. Reno, NV: 7 - 10 January 2008. 